\title{
Abstracts of the 2012 Paramedics Australasia Conference
}

\author{
Hobart, Tasmania, Australia \\ $2^{\text {nd }}$ and $3^{\text {rd }}$ November 2012
}

\section{Conference Presentation Abstracts}

\section{The Relationship between Pre-Hospital Fluid Resuscitation in Hypotensive Persons with Severe Traumatic Brain Injury and Glasgow Outcome Scale Helen Webb}

Australian Catholic University, Ballarat Victoria, Australia

\section{Background}

The objective of this research is to investigate the relationship between pre-hospital fluid resuscitation and functional outcome of persons with hypotension and severe traumatic brain injury.

\section{Methods \\ Data were retrospectively collected from ambulance service patient care records and hospital medical records of 326 patients with severe traumatic head injury. Data was analysed using stepwise linear regression analysis with confidence interval $(\mathrm{Cl})$ set at $95 \%$ for the regression coefficient. Correlation analysis applied included 2-tailed Spearman's rho for categorical variables and 2-tailed Pearson's correlation for continuous variables. Outcome was measured using the Glasgow Outcome Scale (GOS). Independent samples t-test was utilised to compare means. All p-values were set at the $p<$ 0.05 level to be considered statistically significant.}

\section{Results}

A total of 83 patients experienced an episode of pre-hospital hypotension (systolic BP $<90$ $\mathrm{mmHg}$ ), which represented $25.5 \%$ of the total cohort. Patients with severe traumatic brain injury and pre-hospital hypotension who did not receive pre-hospital fluid resuscitation had markedly higher rates of improved outcome than those who did receive fluid resuscitation $32.3 \%$ versus $13.5 \%$ respectively $(p=0.001)$. Hypotensive patients who received pre-hospital fluid resuscitation demonstrated higher mortality $(73.1 \%$ vs $54.8 \%)$ $(p=0.015)$.

\section{Conclusions}

Results were unclear as to the benefit of fluid volume replacement in the pre-hospital setting. Episodes of pre-hospital hypotension were demonstrated as a significant predictor of poorer outcome and linear regression analysis also demonstrated that the presence of hypotension on arrival at the emergency department was a significant predictor of outcome following severe traumatic head injury. However, the administration of fluid volume replacement in the pre-hospital setting was not found to be a significant predictor of outcome of improved outcome.

\section{The Efficacy of Pre-hospital Endotracheal Intubation in Severe Traumatic Brain Injury Helen Webb}

Australian Catholic University, Ballarat Victoria, Australia

\section{Background}

The objective of this research is to investigate the relationship between pre-hospital endotracheal intubation and functional outcome of persons with severe traumatic brain injury.

\section{Methods}

Data were retrospectively collected from ambulance service patient care records and hospital medical records of 326 patients with severe traumatic head injury. Data was analysed using stepwise linear regression analysis with confidence interval $(\mathrm{Cl})$ set at $95 \%$ for the regression coefficient. Correlation analysis applied included 2-tailed Spearman's rho for categorical variables and 2-tailed Pearson's correlation for continuous variables. Outcome was measured using the Glasgow Outcome Scale (GOS). Independent samples t-test was utilised to compare means. All $p$-values were set at the $p<$ 0.05 level to be considered statistically significant.

\section{Results}

A total of 65 patients received endotracheal intubation in the pre-hospital setting. For those patients who received pre-hospital intubation, $95.3 \%$ had a poor outcome (GOS 1, 2 or 3 ) versus $59.1 \%$ for those who were intubated in hospital ( $p<0.001)$. Improved outcomes were noted in $4.6 \%$ of those who were intubated in the pre-hospital setting (GOS 4 or 5 ) and $40.9 \%$ of those intubated within the in-hospital setting $(p<$ $0.001)$. A high mortality rate was also noted in 
patients who received pre-hospital endotracheal intubation versus intubation in the ED $(75.4 \%$ vs $34.2 \%$ respectively) (Spearman's rho $r=0.338, p$ $<0.001,2$-tailed). Stepwise linear regression revealed pre-hospital endotracheal intubation to be a significant predictor of poorer outcome ( $r=-$ $0.433, p<0.001,95 \% \mathrm{Cl}=1.011$ to 1.858$)$.

\section{Conclusion}

Pre-hospital endotracheal intubation was not associated with improved outcome (GOS 4 or 5 ) following severe traumatic brain injury.

\section{Gender bias in 12-lead ECG acquisition from ambulance patients with cardiac-type chest pain; an observational study.} Bronwyn Henderson, Bronwyn Tunnage, Steve Taylor

Auckland University of Technology, Auckland, New Zealand

\section{Background}

Female patients suffering from Acute Coronary Syndrome (ACS) receive fewer investigations and treatments than male patients, in both the prehospital and hospital environments. Failure to acquire an early 12-lead ECG can impact on accessing timely treatment and could be a barrier to women receiving appropriate medical care. Some authors suggest that, because female patients are more likely to present with atypical symptoms, their cardiac diagnosis is missed and consequently they receive different treatment. Unlike previous studies exploring gender bias in investigation of cardiac-type chest pain, this study controlled for the paramedic's working diagnosis. The primary outcome was to establish whether female patients in the ambulance setting, are as likely to receive a 12-lead ECG as a male patient, when the paramedic's working diagnosis is that of ACS.

\section{Methods and Results}

We reviewed 26,273 ambulance patient report forms from four centres in New Zealand spanning a one year period and identified 1,680 forms that met the study inclusion criteria; $52 \%$ were male patients. Overall rates of 12-lead ECG acquisition were low at $45 \%$ of cases. $50 \%$ of males $(n=432)$ had a 12-lead ECG acquired compared with $37 \%$ of females $(n=276)(P<0.001)$. After adjusting for potential confounders, the odds ratio for female patients receiving a 12-lead ECG was $0.50(95 \%$ confidence interval, 0.40 - 0.62) compared with male patients. The probability of having a 12-lead ECG acquired varied significantly between study centres, from $86 \%$ to $30 \%$.

\section{Conclusions}

Even when the paramedic suspected that a female patient was having an acute coronary event, a 12-lead ECG was less likely to be acquired by the ambulance crew, than for a male patient with the same working diagnosis.

Secondary outcomes identified local variation in practice, suggesting the possibility of improving the frequency of this vital investigation in ACS.

\section{Women's attitudes to 12-lead ECG acquisition in the ambulance setting. Rachael Wallen ${ }^{1}$, Bronwyn Tunnage ${ }^{1}$, Susan Wells ${ }^{1,2}$

\author{
${ }^{1}$ Auckland Univerity of Technology, Auckland, New \\ Zealand \\ ${ }^{2}$ University of Auckland, Auckland, New Zealand
}

\section{Background}

While best practice guidelines emphasise early 12-lead ECG acquisition in acute coronary syndrome (ACS), symptomatic female patients are less likely to have this investigation performed by ambulance crew than male patients. This study explores women's attitudes to ECG acquisition in the ambulance setting.

\section{Methods}

Fifty female participants were enrolled in the study aged from 21 to 76 years of age and including seven ethnicities. Participants completed a demographic questionnaire prior to ECG acquisition and, later, a second questionnaire eliciting their attitudes towards ECG acquisition including sex of the paramedic operator and siting of the precordial electrodes. Two standard 12lead ECG were recorded from each participant; one with electrodes V3-V6 placed on the breast tissue and a second with these electrodes located under the breast. Waveform morphology and amplitude and the monitor-provided interpretation were also compared for the two ECG.

\section{Results}

Most participants (96\%) preferred an early ECG in the ambulance setting rather than waiting for hospital admission, even when the ambulance crew was all-male. $52 \%$ of participants preferred having the ECG recorded over the breast, 10\% favoured under the breast and $38 \%$ had no preference. Minor differences in R, S and T wave amplitudes between the paired ECG were observed in $96 \%$ of the pairs, but none would result in different ambulance treatment pathways.

\section{Conclusions}

This is the first study of ECG acquisition and precordial electrode placement in women in the context of ambulance care and barriers to ACS care. Women's choice of an early ambulanceacquired ECG demonstrated that they placed a higher premium on preserving their health than their modesty. Siting precordial electrodes on the breast, rather than under, was preferred by more 
women in this study and appeared to provide an equally accurate ECG.

\section{Incidence of unrecognised oesophageal intubation in patients intubated by paramedics and transported to an Australian metropolitan tertiary hospital. Martin Nichols ${ }^{2,1}$, Joe Acker ${ }^{1}$, Jason C Bendall ${ }^{2,1}$, Stephen Asha ${ }^{3,4}$ \\ ${ }^{1}$ School of Biomedical Sciences, Charles Sturt University, Bathurst, NSW, Australia \\ ${ }^{2}$ Ambulance Service of NSW, Rozelle, NSW, Australia \\ ${ }^{3}$ Faculty of Medicine, UNSW, Kensignton, NSW, Australia \\ ${ }^{4}$ St George Hospital, Kogarah, NSW, Australia}

\section{Background}

To determine the incidence of unrecognised oesophageal intubation by intensive care paramedics in a metropolitan emergency medical service setting.

\section{Methods}

A retrospective observational analysis was conducted within an Australian emergency medical service. An ambulance service database of patient health care records was used to identify patients who had been intubated by paramedics between January 1, 2007 and December 31, 2010 and transported to a tertiary metropolitan emergency department. Medical records were reviewed to determine the position of the endotracheal tube (ETT) on arrival in the emergency department.

\section{Results}

During the study period, 196 patients were identified as having an ETT in-situ on arrival in the ED. There was inadequate documentation for 67 patients to determine ETT placement. Of the 129 patients included in the final analysis, $4(3.1 \%$, $95 \% \mathrm{Cl} 0.9-7.8 \%$ ) had an unrecognised oesophageal intubation. The final ETT positions of the remaining 125 patients were $85.3 \%(95 \% \mathrm{Cl}$ $78.0-90.9 \%)$ located in the trachea, $10.1 \%(95 \%$ Cl $5.5-16.6 \%$ ) located in a primary bronchus, $0.8 \%(95 \% \mathrm{Cl} 0.0-4.2 \%)$ in the larynx and $0.8 \%$, $95 \% \mathrm{Cl} 0.0-4.2 \%)$ in the pharynx.

\section{Conclusion}

The incidence of unrecognised oesophageal intubations in this study was consistent with other reports in the literature, although higher than expected given the training and equipment used in this setting. An incidence of unrecognised oesophageal intubations of zero should be the goal of emergency medical services. The incidence of unrecognised oesophageal intubations may be reduced through recursive training programs and the use of quantitative waveform capnography.

\section{Success rates of paramedic insertion of laryngeal mask airways: A review and critical appraisal of the literature Jason C Bendall}

School of Biomedical Sciences, Charles Sturt University, Bathurst, NSW, Australia

\section{Background}

Variable successful insertion rates have been reported for laryngeal mask airways with results dependant on the setting. Success rates of physician inserted laryngeal mask airways in the peri-operative setting approach $100 \%$. Published infield success rates for paramedics are in the order of $70 \%$ despite much higher success rates observed in manikin studies. There should be no reason why paramedic laryngeal mask insertion rates should not approach those attained in the peri-operative setting. The reasons for lower than expected insertion success rates are likely to be multi-factorial and are likely to involve education, exposure, device and paramedic factors. To identify the reasons for lower than expected prehospital laryngeal mask insertion rates.

\section{Methods}

A literature review will be undertaken of the electronic medical databases Ovid Medline, EMBASE, CINAHL Plus, Cochrane Systematic Review, and Meditext. Articles were included if they reported on success rates of emergency medical services personnel using laryngeal mask airways in real or simulated settings. Articles not written in English were excluded.

\section{Results}

This paper will present the findings of the literature search and will critically appraisal relevant studies. Reasons for lower than expected insertion success rates in the prehospital setting will be explored. Strategies to improve success rates will be discussed.

\section{Conclusions}

Lower than expected success rates pose a clinical risk to patients and rates of successful insertion should be monitored by jurisdictions currently using laryngeal mask airways. Prospective studies are needed to evaluate the effectiveness of various strategies that may improve success rates. 


\section{Do undergraduate paramedics students require prehospital placements with ambulance services prior to graduation? Jason C Bendall}

\section{School of Biomedical Sciences, Charles Sturt} University, Bathurst, NSW, Australia

Anecdotally, institutions providing undergraduate paramedic degrees report difficulty securing prehopsital clinical placements with ambulance services. Additionally, placements within the emergency medical service sectors are of variable durations, variable quality, have variable case mix and case volume and have variable quality of mentorship. It is difficult to locate employer expectations of new graduates with regard to what constitutes 'work ready' and there is industry acknowledgement of the difficulties nationally securing clinical placements (CAA, 2010). An educational model with the end in mind may determine that the overwhelming majority of clinical educational needs of undergraduate paramedics could be met within emergency department, medicine, surgical, critical care, allied health, community and primary care settings. Access to inter-professional mentors (including medical, nursing and allied health) prior to exposure to emergency medical service mentors may increase the breadth and depth of exposure to 'medicine' as it pertains to the practice of paramedicine. Broadening the breadth and depth of clinical exposure supervised by registered health professionals will provide welcome exposure of undergraduates to professional attributes and behaviours. Thinking of my personal journey through vocational paramedic education followed by graduate medical training I feel fortunate to have been exposed to another paradigm of clinical education that has enhanced my paramedic practice. The focus of undergraduate paramedic degree programs should not only be on meeting the needs of employers now, but should be providing paramedics of the future. To achieve this, there will need to be considerable exposure to nonambulance clinical placements with sufficient immersion to actually gain the necessary knowledge, skills and judgement. The synergies between paramedicine and emergency medicine are significant and opportunities to embed undergraduate placements should be explored. This paper will reflect on my undergraduate and postgraduate medical education and the potential relevance of this to the practice of paramedicine.

\section{Undergraduate paramedic students' empathy levels: A two-year longitudinal study \\ Brett Williams ${ }^{1}$, Malcolm Boyle ${ }^{1}$, Richard} Brightwell ${ }^{2}$, Scott Devenish ${ }^{3}$, Peter Hartley, Michael McCall ${ }^{5}$, Paula McMullen ${ }^{5}$, Graham Munro $^{6}$, Peter O'Meara ${ }^{7}$, Helen Webb ${ }^{8}$, Anthony Weber ${ }^{9}$

${ }^{1}$ Monash University, Melbourne, Australia

${ }^{2}$ Edith Cowan University, Perth, Australia

${ }^{3}$ Queensland University of Technology, Brisbane,

Australia

${ }^{4}$ Victoria University, Melbourne, Australia

${ }^{5}$ University of Tasmania, Hobart, Australia

${ }^{6}$ Charles Sturt University, Bathurst, Australia

${ }^{7}$ La Trobe University, Bendigo, Australia

${ }^{8}$ Australian Catholic University, Ballarat, Australia

${ }^{9}$ Central Queensland University, Toowoomba, Australia

\section{Background}

Empathetic behaviour is widely regarded as being important in achieving positive outcomes for patients. Empathetic healthcare attitudes in patient care have been credited with increasing patient compliance, reducing patient stress levels, minimising the rate of medical errors and achieving optimal physiological results. However, whether paramedic students have positive empathetic attitudes is largely unknown. Therefore, the objective of this study was to assess the extent of empathy in paramedic students over a two-year period from six Australian universities

\section{Methods}

This was a cross-sectional study employing a convenience sample of first, second, and third year undergraduate paramedic students during May 2011 and 2012. Student empathy levels were measured using a standardised selfreporting instrument: Jefferson Scale of Physician Empathy - Health Profession Students' version (JSPE-HPS) maximum score 140.

\section{Results}

A total of 1,793 students participated in the study of which $57 \%(n=979)$ were females. The majority of students were aged between $20-24$ years $44 \%$ $(n=757)$ and almost half the students $49 \%(n=856)$ were enrolled in first year. The two-year overall JSPE-HPS mean was 105.92 ( $S D=12.85)$. Females had greater mean JSPE-HPS empathy scores than males $107.45 \vee 103.86(p<0.0001$, $d=0.28$ ). There was a statistically significant difference between mean scores from Monash University, Charles Sturt University and Victoria University participants $(p=0.019, d=0.22)$. Interestingly, JSPE-HPS empathy scores did not decline as students progressed through their degree $(p=0.541)$. 


\section{Conclusions}

Results from this 2-year study provide the paramedic discipline with important empirical evidence in its attempt to better understand the complex construct of empathy. Moreover, this study provides further evidence that females are more empathetic than their male counterparts, and that differences are found between some universities. The fact that empathy scores did not decline through course progression contradicts the international empathy literature; and is worthy of closer examination in future ongoing studies.

\section{The Medical Condition Regard Scale: Measuring attitudes in paramedic Students}

Brett Williams ${ }^{1}$, Malcolm Boyle ${ }^{1}$, Richard Brightwell ${ }^{2}$, Scott Devenish ${ }^{3}$, Peter Hartley ${ }^{4}$, Michael McCall ${ }^{5}$, Paula McMullen ${ }^{5}$, Graham Munro $^{6}$, Peter O'Meara ${ }^{7}$, Helen Webb ${ }^{8}$, Anthony Weber ${ }^{9}$

${ }^{1}$ Monash University, Melbourne, Australia

${ }^{2}$ Edith Cowan University, Perth, Australia

${ }^{3}$ Queensland University of Technology, Brisbane, Australia

${ }^{4}$ Victoria University, Melbourne, Australia

${ }^{5}$ University of Tasmania, Hobart, Australia

${ }^{6}$ Charles Sturt University, Bathurst, Australia

${ }^{7}$ La Trobe University, Bendigo, Australia

${ }^{8}$ Australian Catholic University, Ballarat, Australia

${ }^{9}$ Central Queensland University, Toowoomba, Australia

\section{Background}

Understanding students' attitudes towards certain medical conditions is an important part of the education that students receive prior to employment in the healthcare workforce. One of the key characteristics of any healthcare worker is a non-judgemental, egalitarian attitude, and yet historically, the reactions to particular medical conditions have been often described as hostile, prejudiced, and judgmental. The objective of this study was to assess the attitude toward specific medical conditions of paramedic students from six Australian universities.

\section{Methods}

A cross-sectional study employing a convenience sample of first, second, and third year undergraduate paramedic students during May 2011 and 2012. Student's attitudes toward four medical conditions commonly encountered in the out-of-hospital setting were measured using a standardised self-reporting instrument: Medical Condition Regard Scale (MCRS), maximum score 66.

\section{Results}

A total of 1,793 students participated in the study of which $57 \%(n=979)$ were females. The majority of students were aged between $20-24$ years $44 \%$ $(n=757)$ and almost half the students $49 \%(n=856)$ were enrolled in first year. The two-year mean scores for each of the medical conditions were all < 40: Intellectual disability: $\mathrm{M}=38.67, \mathrm{SD}=8.23$; Substance abuse: $M=35.51, S D=9.17$; Attempted suicide: $M=38.09, S D=9.56$; Acute mental illness: $M=39.29, S D=10.02$. There was a statistically significant difference between mean scores for each medical condition across universities $(p<0.0001)$. Intellectual disability mean scores declined from $1^{\text {st }}$ to final year studies $(p<0.0001)$ while the remaining medical conditions were unchanged. There were no statistically significance differences between genders.

\section{Conclusions}

Results from this 2-year study provide the paramedic discipline with important empirical evidence in its attempt to better understand the complex construct of attitude. The findings from this study found that students reported poor regard for patients with intellectual disability with mean scores declining through their undergraduate program.

\section{Simulation as an inter-professional education tool that puts paramedics, doctors and nurses, in the same school Ann Lazarsfeld-Jensen}

\section{Charles Sturt University, Bathurst, NSW, Australia}

Simulation as a tool of inter professional education (IPE) is a research focus at Charles Sturt University. Scripted scenarios with high fidelity manikins showed the use of IPE as a tool in training needs analysis for GPs and paramedic undergraduates, as well as for the transfer of specialist knowledge in ways that enhance respect and teamwork. IPE paediatric emergency scenarios were developed, trialled and evaluated by paramedic and nursing academics at CSU and then repeated with GP participants. The project was evaluated using focus groups,

questionnaires, video observation and participant feedback. Stressors introduced in the scenario scripts by actors were significant in challenging both working relationships and clinical skills. Medical emergencies are uncommon in doctor's surgeries, but such rare events make strong demands on clinical decision-making capacity as well as little used skills. Unlike paramedics, GPs are not accustomed to working in chaotic public spaces and they have few opportunities to develop and maintain advanced life support skills. Patient safety concerns and the dearth of clinical placements have made simulation based medical education an effective way of enabling students to learn from their errors (Ziv, Ben-David, \& Ziv, 2005). Simulation can be used at multiple levels in teaching, but it is fundamentally used for the safe practice of psycho motor skills that require repetition for mastery (McGaghie, Issenberg, 
Petrusa, \& Scalese, 2010). Simulation in teaching hospitals allows doctors skills to be rigorously evaluated and systematically improved through (Adler, et al., 2009). Inter professional education (IPE) is a more complex emerging field of education in Australia which addresses communication problems between health professional which have been identified as a risk factor in sentinel events. The use of video replays and debriefing allows practitioners to safely explore how they functioned in an unfamiliar and uncertain environment.

\section{Interprofessional perceptions of paramedic students} Brett Williams ${ }^{1}$, Malcolm Boyle ${ }^{1}$, Richard Brightwell' ${ }^{2}$, Michael McCall ${ }^{3}$, Paula McMullen ${ }^{3}$, Graham Munro ${ }^{4}$, Peter O'Meara ${ }^{5}$, Vanessa $W e b b^{1}$

${ }^{1}$ Monash University, Melbourne, Australia

${ }^{2}$ Edith Cowan University, Perth, Australia

${ }^{3}$ University of Tasmania, Hobart, Australia

${ }^{4}$ Charles Sturt University, Bathurst, Australia

${ }^{5}$ La Trobe University, Bendigo, Australia

\section{Background}

Healthcare systems are evolving to feature the promotion of interprofessional practice more prominently. The development of successful and functional interprofessional practice is best achieved through interprofessional learning (IPL). Given that many paramedic programs take an isolative uni-professional educational approach, serious questions must be raised as to whether students are being prepared for the interprofessional healthcare workplace. The objective of this study was to assess the attitudes of paramedic students towards IPL and cooperation across five universities.

\section{Methods}

Using a convenience sample of paramedic students - attitudes towards IPL and cooperation were measured using two standardised selfreporting instruments: Readiness for Interprofessional Learning Scale (RIPLS) 5-point Likert-scale ( $1=$ strongly disagree and $5=$ strongly agree) and Interdisciplinary Education Perception Scale (IEPS) 6-point Likert-scale (1=strongly disagree and $6=$ strongly agree).

\section{Results}

303 students participated in the study (39\% response rate). The majority of students were enrolled in second year $n=149(49.2 \%)$ with most students who participated being female $n=163$ $(53.8 \%)$ and undertaking a single paramedic degree $n=242(79.9 \%)$. Students identified most strongly with the idea teamwork skills were vital for all students to learn (mean=4.58, $S D=0.60$ ). Students agreed most strongly with the statement that individuals in the paramedic profession needed to cooperate with other professions (mean $=5.31, \mathrm{SD}=0.81$ ). Students from CSU, ECU, and $M U$ had a significant effect regarding the RIPLS subscale 'positive professional image' $(p<0.001$, eta $=0.14)$. Students from ECU, and MU had a significant effect regarding the IEPS subscale 'perception of actual cooperation' $(p=0.038$, eta $=0.11)$.

\section{Conclusions}

This study has found no compelling evidence that students' readiness for IPL is significantly affected by either their gender or the type of degree undertaken. In contrast it was seen that the tertiary institutions involved in this study produced students at different levels of preparedness for IPL and cooperation.

\section{Improving Patient Outcomes via Pre- hospital Registries Karen Smith ${ }^{1,3}$, Amee Morgans ${ }^{1,2}$ \\ ${ }^{1}$ Ambulance Victoria, Doncaster, VIC, Australia \\ ${ }^{2}$ Monash University, Prahran, VIC, Australia \\ ${ }^{3}$ University of Western Australia, Perth, WA, Australia}

\section{Background}

Surveillance through a registry, where consecutive patients are enrolled to create complete patient populations, is a powerful tool for describing healthcare, including the complications and benefits of different therapies. A registry can drive a quality agenda and fosters a performance culture. Ambulance services are ideally placed to capture epidemiological data on key patient groups who may not present to other health agencies.

\section{Methods}

The implementation of VACIS, an in-field electronic patient care record and linked clinical database, has facilitated the collection of prehospital data. Using filters from the Ambulance Victoria Data Warehouse key patient groups are identified for inclusion in registries.

\section{Results}

AV maintains the Victorian Ambulance Cardiac Arrest Registry, which captures data on all patients in cardiac arrest attended by ambulance. Data dates back to 1999 and the registry includes $>60,000$ patient episodes. Monitoring of care has resulted in significant survival improvements. The Turning Point (TP) Drug and Alcohol Ambulance Registry captures all metropolitan ambulance attendances to drug and alcohol patients. Surveillance has resulted in identification of aetiological trends and development of specific Clinical Practice Guidelines for drug overdoses. 
AV links pre-hospital data with the Victorian State Trauma Registry on a quarterly basis via probabilistic linkage of DW data with VSTR. The linked data is used to examine adherence to the Trauma Triage Guidelines and monitor the quality of care of major trauma patients.

\section{Conclusions}

AV has established important patient registries through key partnerships. The registries include data on patient populations who would not be captured otherwise (eg cardiac arrest and trauma patients who die at the scene and non-transported overdose patients). Future initiatives include surveillance of diabetic emergencies with Southern Health and the expansion of the TP registry to include rural overdose patients and mental health patients.

\section{Ambulance Victoria Stroke Management Grant Hocking}

Ambulance Victoria, Victoria, Australia

\section{Background}

The Victorian Department of Health released the Stroke Care Strategy for Victoria (2007) and the National Stroke Foundation published Clinical Guidelines for Stroke Management 2010 and the Acute Stroke Services Framework 2011. The strategy states that a person with stroke symptoms should be transported to the nearest health service providing the most appropriate investigations and acute stroke care. The Strategy noted the success of designated trauma centres and indicated that similar improvements in patient outcomes could be achieved in stroke care. The purpose of the study was to improve the number of Ambulance Victoria (AV) patients reaching health services providing emergency stroke services.

\section{Methods}

Changes to align paramedic clinical practice with the Strategy were implemented into the AV Stroke Management Clinical Practice Guidelines (CPG) and supported by paramedic education. The CPG change detailed the revised stroke management process included a one hour timeframe for travel to an appropriate facility and a 4.5 hour window from symptom onset. Facilities offering Stroke Unit Care (SUC) were communicated via a bulletin and updated regularly. Stroke cases transported by AV in non-metropolitan areas prior to the guideline change (2010) were examined to map the case location against the location of health facilities providing SUC and thrombolysis. The data was compared to post guideline change (2011).

\section{Results}

The data identified the CPG change resulted in a change in destination for stroke patients, with a higher proportion $(58.5 \%$ v $78.5 \%)$ reaching SUC and thrombolysis capable facilities within an approved clinical timeframe.

\section{Conclusions}

This study demonstrates translation of research into clinical practice and highlights the importance of updating clinical and operational procedures to reflect current national guidelines. The results show the importance of ambulance services and paramedic clinical decision making in improving patient pathways to highest level care and optimal health outcomes.

\section{Tell me why I don't like Mondays: Time of day, day of week patterns in ambulance demand}

Kate Cantwell ${ }^{1}$, Amee Morgans ${ }^{1,2}$, Paul Dietze $^{3}$, Karen Smith ${ }^{1,2}$, Michael Livingston ${ }^{4}$

${ }^{1}$ Monash University, Melbourne, Victoria, Australia
${ }^{2}$ Ambulance Victoria, Melbourne, Victoria, Australia
${ }^{3}$ Burnet Institute, Melbourne, Victoria, Australia
${ }^{4}$ Turning Point Alcohol and Drug Centre, Melbourne,
Victoria, Australia

Background

People's occupational, social and recreational activities generally occur in distinct time of day, day of week temporal patterns. Certain physiological parameters and disease states also show temporal variation. It is not known if the temporal nature of people's activities, physiological parameters or disease states transfers into patterns of injury or disease that can be observed in ambulance demand. The aim of this study was to examine four years of ambulance events for time of day, day of week (temporal) trends, by case type, age and location.

\section{Method}

This was a retrospective review using electronic patient data from the Victorian Ambulance Clinical Data Warehouse from 2007 to 2011. Cases were sorted into case type categories using ICD coding techniques. Data was analysed using univariate and multivariate statistical techniques.

\section{Results}

Temporal patterns exist in Ambulance workload for various case types. Suspected cardiac arrest cases have distinct geo-temporal trends which are common across the world. Case types involving young adults occur more frequently on weekends but elderly cases occur during the week. Paediatric cases also have distinct temporal distributions. 


\section{Conclusions}

Distinct temporal patterns appear in ambulance demand when cases are sorted by case type, age and location. These patterns have been used by one international ambulance service to decrease response times to cardiac arrest. This may represent a valuable source of knowledge to improve efficiency of local Ambulance services.

\section{Scald burns in children aged 15 and younger in Australia and New Zealand - an analysis of the Bi-National Burns Registry Dorothee Schricke ${ }^{2}$, Paul Jennings ${ }^{1,2}$, Dale Edgar $^{3}$, John Harvey ${ }^{4}$, Heather Cleland ${ }^{5}$, Fiona Wood $^{3}$, Peter Cameron ${ }^{2}$ \\ ${ }^{1}$ Monash University Department of Community Emergency Health and Paramedic Practice, Melbourne, Victoria, Australia \\ ${ }^{2}$ Monash University Department of Epidemiology and Preventive Medicine, Melbourne, Victoria, Australia ${ }^{3}$ Royal Perth Hospital McCombs Foundation of Western Australia, Perth, Western Australia, Australia \\ ${ }^{4}$ The Children's Hospital at Westmeade, Sydney, New South Wales, Australia \\ ${ }^{5}$ The Victorian Adult Burns Unit, The Alfred Hospital, Melbourne, Victoria, Australia}

\section{Background}

Scalds are a common injury in children and a frequent reason for hospitalisation despite being potentially preventable.

\section{Methods}

This cohort study reports on 730 children aged 14 years or younger who sustained a scald between October 2009 and September 2011, and were admitted to a burns unit in Australia or New Zealand. Data were extracted from the Bi-National Burns Registry (Bi-NBR). Summary statistics were used to describe the epidemiology and prehospital/hospital management of the cohort, and logistic regression models were fitted to establish the association of factors with dependant variables.

\section{Results}

Scald injury contributed $56 \%$ (95\% Cl 53-59\%) of all paediatric burns reviewed during the study period. There were two high risk groups identified; male toddlers age one to two, contributing $34 \%$ (95\% Cl $31-38 \%$ ) of all scalds, and indigenous children who were over 3 times more likely to experience a scald requiring admission to a burns unit than their nonindigenous peers. First aid cooling by nonprofessionals is initiated in $89 \%(95 \% \mathrm{Cl} 86-91 \%)$ of cases but only $19 \%(95 \% \mathrm{Cl} 16-23 \%)$ performed as recommended (i.e. cool running water for at least 20 minutes within two hours of injury).

\section{Conclusion}

Scald burns are common in children 14 years and under, particularly in male toddlers. The findings of this study highlight the need to encourage nonprofessionals to deliver effective first aid and reinforce the importance of targeted prevention campaigns that reduce the burden of paediatric scald burns in Australia and New Zealand.

\section{Epidemiology of ambulance responses to older people who have fallen: a prospective observational study. Paul M Simpson ${ }^{1,2}$, Jason C Bendall ${ }^{1}$, Anne Tiedemann ${ }^{2,4}$, Stephen R Lord ${ }^{2,3}$, Jacqueline CT Close 2,3 $^{2,4}$}

\author{
${ }^{1}$ Ambulance Service of New South Wales, Sydney, New \\ South Wales, Australia \\ ${ }^{2}$ Neuroscience Research Australia, Randwick, New \\ South Wales, Australia \\ ${ }^{3}$ University of New South Wales, Randwick, New South \\ Wales, Australia \\ ${ }^{4}$ The George Institute for Global Health, Sydney, New \\ South Wales, Australia
}

\section{Background}

To prospectively describe 1) the epidemiology of older people who fall and call an ambulance; 2) the operational epidemiology of the ambulance responses they receive, and 3) the characteristics of the falls and resulting patient presentations.

\section{Methods}

A prospective observational study of nonconsecutive people aged $\geq 65$ who had fallen and called for an ambulance between October 12010 and June 30 2011. A 'paramedic falls network' of 380 paramedics was created across NSW to recruit patients and collect data, using a special data collection tool created specifically for the study by experts in falls and balance. The data from the special fall-specific tool was linked to ambulance clinical records and dispatch data. Descriptive statistics were generated, including comparisons of proportions where appropriate.

\section{Results}

There were 1,610 cases eligible for analysis. The dispatch software demonstrated a sensitivity of $72 \%$ for identifying falls, and prioritised $58 \%$ of cases as 'urgent'. Patients were predominantly female $(63 \%)$ and community dwelling (83\%). Forty nine per cent had never called an ambulance for a fall, while $17 \%$ had called within the past month. 'Long lies' were uncommon, with $87 \%$ spending less than one hour on the ground. The most common reason for falling was 'loss of balance' (35\%), followed by a simple 'trip'. New injury/pain was documented in $75 \%$ patients, while $57 \%$ presented with 'normal' physiology; $15 \%$ presented with no new injury/pain and normal physiology. The overall non-transport rate 
was $28 \%$, although this varied according to age, fall location and residential status.

\section{Conclusion}

Falls experienced by older people have major operational and clinical implications for ambulance services. This study provides valuable prospective data to support development of new models of care aimed at optimising service delivery to older fallers.

\section{Defining their professional boundaries of community paramedics and measuring their impact on health outcomes. Peter O'Meara ${ }^{1,6}$, Christine Stirling ${ }^{2}$, Judi Walker $^{3,2}$, Daryl Pedler ${ }^{4,3}$, Michel Ruest ${ }^{5}$ \\ ${ }^{1}$ La Trobe University, Bendigo, Victoria, Australia ${ }^{2}$ University of Tasmania, Hobart, Tasmania, Australia \\ ${ }^{3}$ Monash University, Moe, Victoria, Australia \\ ${ }^{4}$ Deakin University, Warrnambool, Victoria, Australia \\ ${ }^{5}$ County of Renfrew, Paramedical Services, Pembroke, Ontario, Canada \\ ${ }^{6}$ Charles Sturt University, Bathurst, NSW, Australia}

\section{Background}

The emergence of more complex professional paramedic roles raises questions related to expectations and whether the desired outcomes can be measured. The aims of this research are to validate the professional boundaries of community paramedics and to identify and evaluate tools for the measurement of their impact on health outcomes.

\section{Methods}

The study is being undertaken in the County of Renfrew, Ontario, Canada where a community paramedic role has emerged in response to demographic changes and broader health system reform. Descriptions of the role and the outcome measurement tools currently employed will be sought from managers. Qualitative data will be collected through a combination of direct observation of practice, informal discussions, and focus groups.

The innovative component of this research will be reference to the Australian rural domains of paramedic practice model and the use of boundary theory to identify and analyze how ambulance services and community paramedics create and maintain new role boundaries and identities.

\section{Results}

Results from this study will provide a picture of the working environment of community paramedics and how their role and that of ambulance services may develop as part of the health system. It will identify and evaluate appropriate health outcome measurement tools that will inform quality improvement processes.

\section{Conclusions}

Ambulance services, funders, paramedics, educators and researchers will use the findings as the basis for informed dialogue, debate and discussion about community paramedic roles and how they can contribute toward improvement of health outcomes.

\section{Evidence Based Initiative: The introduction of ipratropium bromide for the treatment of acute asthma exacerbations in the pre-hospital environment.

\author{
Elizabeth Brown ${ }^{1,2}$, Joseph Cuthbertson ${ }^{1}$ \\ ${ }^{1}$ St John Ambulance, Western Australia, Australia \\ ${ }^{2}$ Edith Cowan University, Western Australia, Australia
}

\section{Background}

This initiative was undertaken to make the delivery of care provided to people with respiratory disorders by St John Ambulance Western Australia more evidence based. The focus was the care delivered to those with acute exacerbations of asthma. A review of empirical evidence was undertaken to ascertain whether ipratopium bromide should be introduced into practice for the treatment of acute asthma exacerbations.

\section{Method}

A review of the literature was undertaken by devising an answerable clinical question and carrying out a reproducible $\mathrm{MeSH}$ search of three bibliographic databases.

\section{Results}

In total nine relevant studies were identified (five studies undertaken on children, three on adults and two systematic reviews of both). Studies on children indicated a reduction in admission rates (four studies), an increase in lung function (four studies), a reduction in the need for further dosages of salbutamol sulphate (one study), a reduction in treatment time (one study) and a decrease in secondary exacerbations (one study). Studies on adults found a reduction in admission rates (four studies), an increase in lung function (four studies) and a reduced need for further treatment (two studies). No studies reported any significant adverse effects.

\section{Conclusion}

The introduction of ipratropium bromide in conjunction with salbutamol sulphate therapy has been proven by the literature to improve clinical presentation and reduce hospital admission rates in both adults and children. The introduction of ipratropium bromide into St John Ambulance 
Service Western Australian paramedics' scope of practice will ensure that the care given to patients with acute asthma exacerbations is evidence based. This evidence based practice initiative is neither costly nor time consuming but has the potential to reduce morbidity and hospital admission rates.

\section{Using an interactive technology medium to facilitate undergraduate engagement in interprofessional learning: A mixed methods study} Vanessa Webb, Malcolm Boyle, Brett Williams

Monash University, Melbourne, Australia

\section{Background}

It has been well established that interprofessional teamwork results in optimal patient outcomes. Interprofessional learning (IPL) aids the development and consolidation of positive healthcare attitudes to facilitate superior delivery of patient-oriented care. To date, limited literature has been published examining the efficacy of web-based IPL. This paper will outline the development and evaluation of an interactive IPL website encompassing a number of medicallybased DVD scenarios. The objective of this study was to examine students' attitudes toward IPL using an interactive IPL website evaluated with a mixed methods approach, utilising a before and after study, then focus groups.

\section{Methods \\ Undergraduate students currently enrolled in one of five health-related courses at Monash University: emergency health (paramedics), nursing, occupational therapy, physiotherapy, and nutrition and dietetics were given access to the website. The Readiness for Interprofessional Learning Scale (RIPLS) was used to measure the students' attitudes towards engaging in IPL before and after viewing the site. Focus groups provided qualitative data assessing if and how the interactive IPL website impacted on student's readiness for IPL.}

\section{Results}

The final sample of students who completed both the before and after questionnaires was $n=44$. The majority of students were enrolled in first year $n=16(36 \%)$ and predominately female $n=39$ $(84 \%)$. Students showed a statistically significant change in their responses in the 'shared learning' subscale from before $(M=25.00, S D=5.26)$ to after $(\mathrm{M}=27.19, \mathrm{SD}=3.92), t(41)=-2.17, p=0.35$ (two tailed). A number of themes emerged from the focus groups such as: learner engagement, learner satisfaction, and benefits over face-to-face activities.

\section{Conclusions}

Results from this study suggest the interactive IPL website was a positive experience for students. Before and after results showed improvements within the area 'shared learning' paradigm, while qualitative results suggest learners were engaged and supportive of IPL.

\section{Knowledge translation: Are the next generation of paramedics agents of change? \\ Amanda Ghirardello and Brett Williams}

Monash University, Melbourne, Australia

\section{Background}

Knowledge translation involves the dissemination and application of scientific research findings into clinical practice. In the healthcare arena, uptake of evidence-based assessment and intervention strategies is aimed at reducing inefficiencies and ultimately improving patient outcomes. However numerous studies have purported gaps in knowledge translation in the healthcare professions. Therefore the objective of this study was to assess the extent of knowledge translation in paramedic students at a large Australian university.

\section{Methods}

A cross-sectional study of students across all undergraduate years from Emergency Health and Emergency Health/Nursing was completed. Student knowledge translation levels were measured using the 17-item paper-based Practice Style Inventory (PSI).

\section{Results}

A total of 266 students participated in the study of which $66 \%$ were females. The majority of participants were $<26$ years of age $(n=128)$ and enrolled in second year studies $(n=134)$. Two subscales produced statistically significant differences: 'Evidence/Experience' (extent to which scientific evidence rather than authority is perceived as the best source of knowledge), and 'Nonconformity' (degree of comfort with clinical practices that are out of step with recommendations of leaders). There was a statistically significant difference between gender on the 'Evidence/Experience' subscale $(p=<0.0001, d=0.51)$, and between year levels on the 'Nonconformity' subscale $(p=<0.007, d=-0.63)$.

\section{Conclusions}

This study identified several differences in knowledge translation subscales in the undergraduate paramedic cohorts. Further investigation is warranted in order to better understand barriers and facilitate improved uptake of evidence-based research into clinical practice and ultimately improve patient outcomes. Future 
research using a longitudinal study design to capture changing attitudes to knowledge translation in the postgraduate population may also prove valuable.

\section{Does patient outcome improve when treated by a MICA paramedic over a regular paramedic during cardiac arrest? Alexandra Spence, Ben Rolinson, Eliot Walton, Claire Harrison, Claire Scarlett, Mitchell Pitt}

LaTrobe University, Bendigo, VIC, Australia

\section{Background}

The purpose was to research and appraise journal articles relating to pre hospital patient outcomes in cardiac arrest and we comparised the interventions of MICA paramedics and regular paramedics. This intervention interested us as we wanted to investigate the theory behind the introduction of MICA paramedics in Ambulance Victoria.

\section{Methods}

To find and appraise articles we used a five-step evidence-based practice approach. We created a structured question using the PICO model: Patient, Intervention, Comparison Intervention, and Outcome. This helped determine a range of search terms. We also considered the need to use alternative terms accounting for the lack of consistency in emergency medicine terminology. For example, the American equivalent of MICA paramedics are referred to as 'Advance Life Support Paramedics'. We used the databases CINAHL and PubMed, and Boolean operators such as 'and' and 'or' to find relevant articles. When appraising the articles we examined the design, outcomes, results, and limitations.

\section{Results}

After initially discovering ten articles we found three articles that best suited our needs. Le May et al. 2006 concluded that paramedics who have attained an Advanced Care Paramedic diploma can be trained to accurately interpret the prehospital ECG for diagnosis of STEMI. Woodall et al. 2007 presented that an intensive care paramedic at a cardiac arrest had a positive impact to out of hospital discharge, and a systematic review completed by Irola et al. 2010 supported advanced life support intervening in a case of cardiac arrest.

\section{Conclusions}

The evidence strongly suggests that MICA, Advanced Life Support and/or Intensive Care paramedics improve survival in pre hospital cardiac arrest patients. We support Ambulance Victoria's decision to widely introduce MICA paramedics and believe that patient outcomes will continue to justify their employment.

\section{The ABCs of Adolescence: Recommendations for Ethical Treatment of Adolescents in the Pre-Hospital Environment \\ Suzanne Hocking}

Whitireia Polytechnic, Porirua, New Zealand

\section{Background}

This literature review proposes a set of management protocols for adolescents in the prehospital environment through a discussion of legal and ethical principles as they pertain to these patients.

\section{Methods \\ Information was obtained using medical, psychiatric and legal textbooks published within the last ten years, and corroborating data was collected from online academic databases and other credible sources. Search terms included adolescence, competence, developmental research, help-seeking, maturity, pre-hospital, rapport, self-harm, and staff attitudes.}

\section{Discussion}

Current research increasingly advocates for adolescents to be considered a separate demographic, not only sociologically but also medically, as their brains operates on a different level than that of either adults or paediatrics. Therefore, adolescents should be granted the courtesy of an individualised model of care. The proposed ABCs of Acceptance, Boundaries and Continuity of Care aim to preserve patient autonomy, competence, consent, confidentiality, and nonmaleficence for adolescent patients. By using them as a guide, emergency staff can keep abreast of psychiatric advances and perform patient-focused best practice without letting subjective assessments and attitudes pre-judge behaviour that is actually rooted in developmental processes. Current research around consent emphasises the individual's ability to understand and make reasoned judgements over the criteria of chronological age, making national ambulance guidelines biased against under-16s. Research also shows a desire in medical personnel for improved training around care of adolescents. Such training is proving to be the determining factor in terms of effectiveness of treatment, resilience to negative behaviour, and positive attitudes in staff. Interacting with adolescents objectively and individually based on standardised criteria will allow ambulance staff to equalise quality of care and minimise bias across all age groups.

\section{Conclusion}

There is a clear place for developing a focused set of guidelines for emergency staff when interacting with adolescents based around the ABCs of Acceptance, Boundaries, and Continuity. 
Paramedic Assessment and Referral to Access Medical Care at Home: a randomised trial

Steve Johnston ${ }^{1}$, Moira Sim ${ }^{1}$, Glenn Arendts ${ }^{1}$, Margaret Giles ${ }^{1}$, Toni Wain ${ }^{1}$, Joe Cutbertson ${ }^{2}$, Joanna Smith ${ }^{1}$, May Wong ${ }^{1}$, Russell McKenney ${ }^{1}$, Richard Brightwell ${ }^{1}$

${ }^{1}$ Edith Cowan University, Perth, Western Australia, Australia

${ }^{2}$ St John Ambulance (WA), Perth, Western Australia, Australia

\section{Background}

The aim of the project was to test a paramedic referral pathway to reduce costs while maintaining or improving the quality of care for patients with low-acuity illness or injury who call an ambulance in the Metropolitan Area. A randomised controlled trial was established in partnership with St John Ambulance Australia (WA) Inc. (SJAWA) and Silver Chain's Home Hospital Service (SCHH) to test the intervention, which could reduce pressure on emergency department (ED) services and contribute to ambulance resource availability.

\section{Methods}

Patients who were assessed as suitable by paramedics were randomised to for standard care (control) or referral to Silver Chain Home Hospital's Priority Response Assessment team (intervention), which provides a broad range of clinical services within four hours and treat patients in their place of residence.

\section{Results}

A total of 160 patients were recruited: 78 in the control and 82 in the intervention group and these were used for the analysis of cost, safety and satisfaction outcomes.

\section{Conclusions}

This study demonstrated a reduction in emergency department visits, hospital bed days and ambulance paramedic time with patient in the intervention cohort. In terms of cost benefit analysis projected costs savings for using this intervention gave a significant return on investment. All respondents who were referred to $\mathrm{SCHH}$ were satisfied with the service and would be willing to be treated by $\mathrm{SCHH}$ again.

This study demonstrates the potential cost effectiveness, safety and patient satisfaction with a paramedic referral model in a metropolitan area. We recommend further investigations into paramedic stay and treat models verses referral models.

\section{Paramedic Health Promotion: Connecting Ambulance Services to Communities Buck Reed}

Ambulance Service of NSW, Sydney, New South Wales, Australia

\section{Background}

Health promotion is a vast discipline involving empowering the community to improve its health. Health promotion has traditionally been the domain of specialist health promotion workers or health professionals working in the community context. Paramedics have a unique role in providing health care to the community outside of traditional health facilities and in lower workload areas, such as rural communities, may have significant capacity to participate in non-traditional activities such as health promotion.

\section{Methods}

A literature search was conducted to determine the current level of paramedic engagement in health promotion and the suitability of paramedics to work in the health promotion sphere. The suitability of paramedics undertaking health promotion activities was considered against the framework of current ambulance practice and the needs of health promotion activities.

\section{Results}

Ambulance services operate in a largely responsive paradigm and have rarely focused on preventative health activities other than service promotion and awareness. Within the deeper context of health promotion, there is scope for paramedics to participate these kinds of activities especially in smaller rural communities.

Paramedics are particularly comfortable operating in the community context and this combined with highly developed communications skills, public trust and potential capacity when not performing primary response roles makes them well suited to health promotion activities. Health promotion activities provide an excellent opportunity to partner with other health professionals to provide an interdisciplinary approach to health promotion. It is also likely that increased exposure to paramedics will support core ambulance functions by building relationships between paramedics and their communities and reinforcing the role paramedics play in health emergencies.

\section{Conclusions}

Paramedics can play a key role in improving health in the community through a range of health promotion activities. Paramedics engaging in health promotion supports core roles as well as improving the health of the community. 


\section{Preliminary Development and Testing of a Screening Tool for Review of Paramedic No-Transport Decisions \\ Toby Keene, Megan Davis, Carol Brook}

ACT Ambulance Service, Canberra, Australia

\section{Background}

Cases in which a patient is assessed by Paramedics and not transported to hospital represent a significant clinical risk for ambulance services. Ambulance Services routinely perform retrospective reviews of patient care records to determine whether cases meet organisational standards and identify potential adverse events. The sheer number of cases precludes review of all cases; so many ambulance services rely upon random sampling or limited occurrence screening to identify cases for review. Trigger tools have been used by healthcare organisations to identify potential adverse events from large volumes of patient records.

The Transport Not Required Risk Screening Tool (TRIST) is a trigger tool that screens ambulance electronic patient care records and grades them as potentially high, medium or low risk. TRIST considers 10 different variables, consisting of physiological and operational parameters, resulting in a score from 0 to 30 .

\section{Methods}

This is an exploratory study that aims to assess the validity and reliability of TRIST to accurately screen and identify cases that are high risk for adverse events.

TRIST electronically screens and scores all cases in which a Paramedic has assessed a patient and the patient was not transported to hospital, and grades them as high, medium or low risk. A stratified random sample of cases is selected to undergo a structured clinical review by a Clinical Reviewer in accordance with established organisational review procedures. The reviewer is blind to the TRIST score, and inter-rater reliability will be calculated.

At time of submission, data collection is ongoing. The study aims to review 100 TRIST cases in this exploratory study.

\section{Results}

Results are pending at this time.

\section{Conclusions}

This study aims to develop a trigger tool that will identify ambulance cases at risk of an adverse event.

\section{An investigation into the use of Patient Management Plans (PMPs) by Wellington Free Ambulance (WFA) in New Zealand for repeat ambulance users}

Sean Thompson ${ }^{1}$, Mark Bailey ${ }^{2}$

${ }_{2}^{1}$ Wellington Free Ambulance, Wellington, New Zealand

${ }^{2}$ Whitireia Polytechnic, Wellington, New Zealand

\section{Background}

High demands on ambulance services due to repeat ambulance users represent a significant burden on resources. Few areas of healthcare are exempt from these patients who are commonly, if insensitively, termed "frequent flyers". This New Zealand study investigated the system of patient management plans (PMPs) used by Wellington Free Ambulance (WFA) to address the issues involved with repeat ambulance users and reviewed ways to enable management plans to be effective.

\section{Methods}

Monthly ambulance usage before and after the implementation of PMPs for repeat ambulance users was analysed.

\section{Results}

100 individuals were identified as repeat ambulance users. Three individual patients and their PMPs were specifically analysed.

Patient 1 , a 30 year old female, generated an average of seven calls per month over the five months prior to implementation of plan. This reduced to three calls per month over 15 months following implementation, saving NZ\$3,252.29 per month.

Patient 2, a 56 year old male, generated an average of 11 calls per month over seven months, decreasing to once per month over the seven months following plan implementation, saving $\mathrm{NZ} \$ 6,136.40$ per month.

Patient 3 , an elderly male, generated an average of three calls per month in 2011 and eight calls per month in 2012. His PMP should save up to $\mathrm{NZ} \$ 5,031.84$ per month if successfully implemented.

\section{Conclusions}

From our study of repeat ambulance users, the implementation of PMPs was found to be an effective way to reduce call-out frequency and expenditure. To ensure optimum efficacy of PMPs, the plans should encompass multidisciplinary collaboration, good communication between involved parties, consistent implementation and effective dissemination. PMPs should be individualised, structured yet adaptable, and able to be audited. 
Considering the medical, financial and sociological implications for ambulance service provision, we conclude that the use of effective PMPs should be developed by ambulance services.

\section{The use of Continuous Positive Airway Pressure for management of Acute} Respiratory Failure in the COPD Patient: Time for Pre Hospital Intervention Daniel Cudini ${ }^{1,2}$, Tim Sandford ${ }^{1}$, Matthew Naughton $^{3,4}$

\footnotetext{
${ }^{1}$ Ambulance Victoria, Melbourne, Victoria, Australia ${ }^{2}$ Monash University Department of Community Emergency Health and Paramedic Practice, Frankston, Victoria, Australia

${ }^{3} \mathrm{Head}$ of the General Respiratory and Sleep Medicine Service, Department of Allergy, Immunology and Respiratory Medicine, Alfred Hospital, Melbourne, Victoria, Australia

${ }^{4}$ Monash University Department of Medicine, Alfred Medical Research and Education Precinct, Alfred Hospital, Melbourne, Victoria, Australia
}

\section{Background}

Non invasive ventilation (NIV) in the form of $\mathrm{Bi}$ level Positive Airway Pressure is commonly accepted as the most effective evidence based intervention for the management of patients experiencing Acute Exacerbations of Chronic Obstructive Pulmonary Disease (AECOPD). The pre hospital use of NIV in the form of Continuous Positive Airway Pressure (CPAP) is less well studied. However, current evidence and practice has demonstrated positive outcomes when CPAP is used for the management of AECOPD and Acute Respiratory Failure (ARF). The objective of this study was to determine the optimal CPAP pressure setting $\left(\mathrm{cm} \mathrm{H}_{2} \mathrm{O}\right)$ for the treatment of AECOPD and to identify the pre hospital implications of CPAP application for the management of AECOPD.

\section{Methods}

A comprehensive review of the literature was undertaken using a variety of electronic databases from 1980 to the end of 2011. Inclusion criteria were literature relating to the use of NIV in the form of CPAP to treat patients presenting with AECOPD and Acute Respiratory Failure (ARF). Literature relating to the use of NIV other than in the setting of AECOPD and ARF were excluded.

\footnotetext{
Results

The initial search strategy highlighted 421 potential articles for review, with 106 relevant to the inclusion / exclusion criteria. Pre hospital CPAP used in setting of AECOPD was found to be safe and effective in reducing need for intubation, work of breathing and improvement in other physiological parameters. A starting
}

pressure of $7.5 \mathrm{~cm} \mathrm{H}_{2} \mathrm{O}$ with the capacity to increase pressure to $10 \mathrm{~cm} \mathrm{H}_{2} 0$ was deemed safe and effective. A robust set of criteria for determining the presence of pre hospital ARF, subsequent application of CPAP and contraindications / criteria for failing NIV was also determined from the literature review.

\section{Conclusion}

Sound evidence exists supporting the safe and effective use of pre hospital CPAP for the management of AECOPD.

\section{Paramedic performed fascia iliaca compartment block (FICB) for femoral fractures: a randomised controlled trial Paul McRae ${ }^{1,2}$, Jason C Bendall ${ }^{1,2}$, Veronica Madigan $^{1}$, Paul M Middleton ${ }^{4,3}$ \\ ${ }^{1}$ School of Biomedical Sciences, Charles Sturt University, Bathurst, NSW, Australia \\ ${ }^{2}$ Ambulance Service of NSW, Rozelle, NSW, Australia \\ ${ }^{3}$ Discipline of Emergency Medicine, University of Sydney, Camperdown, NSW, Australia \\ ${ }^{4}$ School of Public Health \& Community Medicine, UNSW, Kensington, NSW, Australia}

\section{Background}

The aim of this study was to evaluate the effectiveness of paramedic performed facia iliaca compartment block (FICB) in the prehospital setting on adult patients with suspected femoral fractures.

\section{Method}

This study was a prospective randomised controlled trial conducted within a prehospital emergency medical service located in regional NSW Australia. Twenty four adult patients with suspected femoral fracture were administered morphine $(0.1 \mathrm{mg} / \mathrm{kg})$ prior to allocation. Patients were randomised to either fascia iliaca compartment block with lignocaine and adrenaline using anatomical landmarks (11 patients) or standard care (further aliquots of intravenous morphine) (13 patients).

\section{Results}

There was evidence that patients treated with FICB had a greater reduction in their median verbal numerical pain score than patients in the standard care group ( $50 \%$ versus $22 \%, z-2.25$, $p=0.025$ ). There was no significant difference in median pain scores between the FICB group and the standard care group on arrival at ED or at the time of triage $(p=N S)$. At the time of transfer from the ambulance stretcher to the hospital bed, there was strong evidence that patients in the standard care group had higher median pain scores than patients in the FICB with patients in the standard care group $(p=0.006)$. There were no adverse 
events directly attributed to paramedics performing the FICB procedure.

\section{Conclusions}

This study found that in adults with suspected femoral fractures in the out-of-hospital setting who received FICB performed by a paramedic had a lower pain score 15 minutes following the procedure and reduced pain during transfer from the ambulance stretcher to the ED bed. Intensive care paramedics appear able to successfully perform FICB in the field. Paramedic performed FICB may offer patients an adjunct to or an alternative to parenteral analgesia for femoral fractures especially in patients at high risk of complications from these agents.

\section{Paramedics as the legal decision maker for patients lacking capacity: Paramedic knowledge of the law, futility and end of life decision making.}

\section{Brian Sengstock}

\section{University of the Sunshine Coast, Sippy Downs, Queensland, Australia}

Over 8600 deaths occurred in Queensland, New South Wales and Victoria as a result of sudden cardiac arrest in the emergency out of hospital setting in 2010. In the absence of a legally appropriate substitute decision maker, or an advance directive, paramedics become the legal decision maker for the patient and are placed in a situation where they must decide what is in the patient's best interests. Statistics indicate that paramedics withhold the provision of lifesustaining treatment in an average of $56.57 \%$ of cases involving sudden cardiac arrest. These decisions, informed by the patient's clinical presentation, are made by paramedics as the legal decision maker as the patient lacks the capacity to consent to the withholding of lifesustaining treatment. Such decisions, whilst based on clinical presentations, can only be lawful if made under certain conditions.

Withholding life-sustaining measures has grave consequences for the patient as their life is ended, potentially exposing paramedics and ambulance authorities to legal action. How an adult's life comes to an end is increasingly subjected to public scrutiny. The extent to which paramedics understand their role as a legal decision maker for patient's lacking capacity is largely unknown. Similarly, paramedic understanding of the law in relation to end of life decisions is largely unknown. What is known is that the law is complex and ambiguous and that this complexity and ambiguity potentially lead to paramedics making an incorrect legal decision to withhold life-sustaining measures.
This paper aims to provide paramedics with an understanding of the legal complexities involved with the decision to withhold life-sustaining measures in the event that the patient lacks capacity. The paper provides an overview of the similarities and differences between the law in Queensland, New South Wales, Victoria and Tasmania, highlighting the key points which paramedics should consider from a legal perspective.

\section{Early termination of EMS resuscitation from out-of-hospital cardiac arrest in Victoria, Australia. Ziad Nehme ${ }^{1,2}$, Emily Andrew ${ }^{1}$, Karen Smith ${ }^{1,3}$ \\ ${ }^{1}$ Ambulance Victoria, Doncaster, Victoria, Australia \\ ${ }^{2}$ Monash University, Prahran, Victoria, Australia \\ ${ }^{3}$ University of Western Australia, Perth, Western Australia, Australia}

\section{Background}

An attempt at resuscitation by emergency medical services (EMS) from out-of-hospital cardiac arrest (OHCA) is defined by the Utstein template as an attempt at CPR or defibrillation. While this population is universally reported in survival statistics, few authors have described the frequency and impact of prematurely terminating care from $\mathrm{OHCA}$.

\section{Methods \\ We retrospectively extracted patient data from the Victorian Ambulance Cardiac Arrest Registry (VACAR) for all adult OHCA where EMS attempted resuscitation between 2003 and 2011. Victims of OHCA were stratified in five minute interval groups according to the duration of resuscitation attempted by EMS. The proportion of attempted resuscitations in each group was compared by year using the chi-square test for equal proportions. Predictors associated with the early termination of a resuscitation attempt $(\leq 10$ minutes) were described using logistic regression.}

\section{Results}

From 2003 to 2011 there were 15,862 adult OHCA where EMS attempted resuscitation, of which $63 \%$ died on scene $(n=9,955)$. The proportion of EMS attempted resuscitation terminated prematurely ( $\leq 10$ minutes) was $11 \%$ in 2003 compared with 19\% in 2011 ( $p<0.001)$. Reasons for early withdrawal of care were documented as: paramedic evidence of prolonged downtime $(55 \%)$, family wishes $(22 \%)$, and first responder resuscitation ceased after ambulance arrival (19\%). Early termination of resuscitation from OHCA was independently associated with a non-cardiac aetiology (OR 1.79, 95\% Cl 1.592.01 ), increased age in years (OR $1.02,95 \% \mathrm{Cl}$ 1.02-1.03), female gender (OR 1.16, 95\% Cl 1.051.28), rhythm of arrival not VF/VT (OR 5.62, 95\% 
Cl 4.72-6.68) and prolonged downtime (OR 3.29, $95 \% \mathrm{Cl}$ 2.98-3.63). In 2011, survival to hospital for OHCA increased from $32 \%$ to $39 \%$ after selectively excluding patients with an early terminated resuscitation attempt.

\section{Conclusion}

As many as one in five EMS attempted resuscitations are prematurely terminated within 10 minutes, and has a significant effect on survival to hospital statistics.

\section{Private providers, public health and paramedic professionalism.}

Ruth Townsend

\section{Australian National University, ACT, Australia}

This paper is part of a legal $\mathrm{PhD}$ looking at paramedic regulation. The objective of the research is to examine the current legislation, and absence of same, that regulates and impacts on paramedic service provision in both the public and private sectors in Australia. The method includes a comparative analysis with two international providers of paramedic services and an analysis of the Australian cross-jurisdictional legislation and policy issues relevant to the area. The results presented in this paper will outline the impacts identified in the comparative analysis and will include a discussion of the impacts of professionalising the paramedic workforce in an international jurisdiction, and more specifically will discuss the change in public expectations and accountabilities of that workforce, along with a discussion of the data gathered on sanctions made against paramedics who are able to be held to different level of account under a national health practitioner registration scheme. This, along with the increase in private sector involvement in the industry, will be considered as a matter of public policy with regard to health workforce development, calls for an increased scope of practice and stronger regulation for paramedics and their services. The results will also examine any relevant differences between public and private providers of services and the way in which policy has been developed to promote the successful development of service provision in both sectors and any shortfall in considering the progress of that development. In short, the paper will offer some insight into the effects of changes to regulation that serves to improve the legal and professional status of paramedics and more strongly regulate the provision of paramedic services by both public and private providers.

\section{Paramedic Practice and the Cultural and Religious needs of Pre-Hospital Patients in Victoria \\ Peter Hartley}

Victoria University, Victoria, Australia

\section{Background}

Religion and culture can impact profoundly on healthcare practices and health outcomes. The Australian community is rich and diverse in differing cultures and religions, and at times of medical emergency the paramedic increasingly will be required to respond to healthcare needs of this diverse community. This study is designed to investigate current paramedic practices as they relate to an awareness of the cultural and religious needs of community groups as a holistic approach. It also incorporates the voices of these community groups from their experiences with emergency paramedics during pre-hospital health care for those living in Melbourne, Australia.

\section{Methods}

Narrative Enquiry and Thematic analysis was used to identify common threads in responses from two independent volunteer groups: community group $(n=20)$ incorporating representatives from the Africa, Asian, Middle Eastern, Muslim, Jewish and Indigenous Australian communities, and paramedic practitioners $(n=20)$. The purpose and nature of and satisfaction with the paramedic callout were investigated using semi-structured interviews during a 90 minute focus group with the individual community groups and individual face-to-face 30 minute interviews with the paramedic group.

\section{Results}

Themes indicate that the paramedics had both a deficit of knowledge and a presence of incorrect knowledge on cultural practices that impact directly on professional practice. In relation to suggestions from the community group participants to ambulance paramedics, communication and respect were the strongest themes. Whilst the paramedics acknowledged the importance of cultural and religious values and preparedness in pre-hospital practice, themes of frustration and increased anxiety as well as a lack of useful cross-cultural education and training were consistent throughout.

\section{Conclusion}

This study recommends strategies to improve interventions with diverse community groups in the pre-hospital setting, and identifies areas of cross-cultural curricula that should be incorporated into paramedic education, and provides a model for a culturally responsive paramedic. 


\section{Violence Against Paramedics in Australia} and the U.S.

Brian Maguire ${ }^{1}$, Peter O'Meara ${ }^{2}$, Barbara O'Neill', Richard Brightwell'

${ }^{1}$ Central Queensland University, Rockhampton, QId, Australia

${ }^{2}$ La Trobe University, Bendigo, Vic, Australia

${ }^{3}$ Lawrence and Memorial Hospital, New London, Ct., U.S.A.

${ }^{4}$ Edith Cowan University, Joondalup. Western Australia., Australia

\section{Background}

In Australia, the rate of occupational injuries among paramedics is eight times higher than the national average for all workers in Australia. In the U.S. the rate of occupational injuries for paramedics is seven times higher than the national average for all workers in the U.S. The Background of this research were to quantify the risk of occupational assault against paramedics and compare national and local data sources.

\section{Methods}

We use both national and local data from both countries to create a broader perspective of the risks of assault among paramedics. All ambulance personnel are collectively referred to herein as paramedics.

\section{Results}

In Australia, national data show 10 to 15 serious assault injuries among paramedics per year. During the same period, one Minister for Health reported that the number of assault cases against paramedics averaged 102 per year in one of the states in Australia alone. In the U.S., the national data indicate a paramedic occupational assault rate of 5.2 cases per 10,000 workers per year; agency level data indicate a rate of 60 cases per 10,000 workers per year.

\section{Conclusions}

The data from both countries indicate a potentially ten-fold difference in findings between national and local sources. This discrepancy reemphasizes the importance of using agency level numerator and denominator data; data that are typically owned by ambulance agencies. The next steps must be done in partnerships between universities and ambulance agencies. These steps include data analysis, case reviews, employee interviews, and the development, implementation and testing of interventions to mitigate the risk of assaults.

\section{Are paramedic students exposed to workplace violence during clinical placements? \\ Malcolm Boyle}

Monash University, Department of Community Emergency Health and Paramedic Practice, Frankston, Victoria, Australia

\section{Background}

Anecdotally we know that paramedic students will not report, in writing, acts of workplace violence during clinical placements as they do not want to jeopardise their opportunity of getting a job. In turn, the ambulance service is reluctant to act upon workplace violence that occurred during the clinical placement unless it is in writing.

Internationally, no study has been published that has investigated the exposure of undergraduate paramedic students to workplace violence. The objective of this study was to identify the type of workplace violence experienced by undergraduate paramedic students and their response to the violence.

\section{Methods}

The study used a cross-sectional methodology in the form of a paper-based questionnaire to elicit undergraduate paramedic responses to workplace violence whilst on ambulance clinical placement. We used the existing Paramedic Workplace Violence Exposure Questionnaire. Students enrolled in the Bachelor of Emergency Health (BEH), Bachelor of Emergency Health/Bachelor of Nursing (BEH/BN) courses at Monash University were eligible to be included in the study.

\section{Results}

Overall response rate was $46 \%$ for the $\mathrm{BEH}, 15 \%$ for the BEH/BN courses. There were $40 \%$ and $21 \%$ males in the $\mathrm{BEH}$ and $\mathrm{BEH} / \mathrm{BN}$ respectively. Average age of students was 24.3 years, range 18 to 47 years, median age was 23 years. The main form of workplace violence was verbal abuse $(19 \%)$ and intimidation (8.3\%) with the male patient/relative commonly the perpetrator. The student's common response was to discuss the incident with family/friends or discuss with another student. They were more likely to "do nothing" and not report the incident to a supervisor.

\section{Conclusions}

These findings suggest there needs to be an anonymous reporting system for students who experience acts of workplace violence whilst attending clinical placements. The establishment of an anonymous reporting system may encourage students to report the violent acts and not "do nothing" about it. 
Do first year paramedic have preconceived attitudes about patients with specific medical conditions? A four-year longitudinal study

Stuart Howard, Chloe Eloise-Verhees, Malcolm Boyle, Brett Williams

Monash University, Melbourne, Australia

\section{Background}

Certain medical conditions have long been associated with stigma, stereotypes, and negative attitudes. These poor attitudes toward patients with specific medical conditions are a major impediment in the provision of healthcare. The objective of this study was to determine the attitude of first year students entering a paramedic course over 4 consecutive yearly (2008-2011) intakes toward patients with intellectual disability, substance abuse, attempted suicide, and acute mental illness.

\section{Methods}

A cross-sectional study using a convenience sample of first year undergraduate paramedic and nursing/paramedic students was used. The students' attitude towards four medical conditions commonly encountered in the prehospital setting was assessed using the Medical Condition Regard Scale (MCRS). The MCRS ranges from 11 to 66 .

\section{Results}

There were 230 students who participated in the study of which $66 \%$ were female with $76 \%$ of all students $<21$ years of age. Students showed the most negative attitudes towards substance abuse, mean $35(S D=6.6)$, and the most positive attitude toward intellectual disability, mean $38.2(S D=6.3)$. The mean attitude towards attempted suicide and mental illness was $37.1(\mathrm{SD}=7.4)$ and $38.1(S D=6.8$ ) respectively. Students in the 2008 cohort displayed significantly higher regard for all medical conditions compared to ensuing years $(p<0.05)$. There was a significant difference in attitude towards attempted suicide in students from the paramedic course compared to those from the nursing/paramedic course, mean 37.9 vs. $35.8(p=0.043)$. Paramedic students also showed a higher regard for mental illness compared with their nursing/paramedic student counterparts, mean 39 vs. mean $36.4(p=0.006)$.

\section{Conclusions}

The study findings suggest that students entering an undergraduate paramedic or paramedic/nursing course have a relatively poor attitude towards the four specific medical conditions, particularly substance abuse. These findings highlight the need to educate students early in their course around these medical conditions.

\section{Prehospital femoral neck fracture management - A review of the literature Samuel Perillo and Malcolm Boyle}

Monash University, Department of Community Emergency Health and Paramedic Practice, Frankston, Victoria, Australia

\section{Background}

Falls are a common incident in the prehospital setting with a significant number resulting in fractures to the neck of femur. There is some disagreement as to the best way to manage these patients, especially the immobilisation of the affected leg. The objective of the study was to identify the best practice management of patients with a fractured neck of femur.

\section{Methods}

A literature search was conducted using medical electronic databases, Medline, EMBASE, and Cochrane Central Register of Controlled Trials (CENTRAL). These databases were searched from the beginning of each database until the end of April 2012. Search keywords included prehospital, EMS, EMT, out-of-hospital, first aid, military medicine, hip fractures and femoral neck fractures. Articles were included if they reported the prehospital management of a patient with a fractured neck of femur. Non-English and hospitalbased articles were excluded. References from articles retrieved were reviewed to ensure potentially relevant articles were not missed

\section{Results}

There were 49 articles located by the search with four articles meeting the inclusion criteria. Following further review of the four articles, three investigated pain management whilst the final one investigated acupressure to decrease anxiety and pain. For the pain studies, one investigated the use of TENS, whilst the other two studies investigated the rate and effectiveness of pain relief. There were no studies that investigated the immobilisation of a femoral neck fracture.

\section{Conclusions}

This study found a lack of evidence for the management of prehospital femoral neck fractures. Further prehospital studies are required to determine the most effective method to immobilise a femoral neck fracture. 
Paediatric TBI screening tools: Can they be adapted to the out-of-hospital setting? Chloe-Eloise Verhees, Stuart Howard, Ben Meadley, Brett Williams

Monash University, Melbourne, Australia

\section{Background}

Traumatic brain injuries (TBI) are a leading cause of death and disability in the paediatric population. In Australia, incidences of mild, moderate and severe paediatric head trauma is estimated at $740-770$ per 100,000 per annum. As such, a number of paediatric TBI clinical screening tools have been developed to help hospital-based clinicians in their decision making process. The objective of this paper is to investigate current evidence for paediatric TBI screening tools.

\section{Methods}

An electronic database was conducted searching key database, Ovid Medline, EMBASE, and CINAHL from their commencement to May 2012. Keywords used in the search criteria included paediatric, pediatric, head, trauma, and out-ofhospital with inclusion criteria consisting of human studies, written in English, paediatric assessment, and screening tools, clinical decision rules, within the paediatric population group and head injury.

\section{Results}

A total of 2547 articles were located, 14 meeting the inclusion criteria and a following 5 articles were located in a review of the reference list revealed 5 more articles meeting the inclusion criteria, producing a total of 19 articles. Four clinical screening tools tested in more than one cohort group are represented within these articles. They include Paediatric Emergency Care Applied Research Network (PECARN), Children's Head Injury Algorithm for the Prediction of Important Clinical Events (CHALICE), National Emergency X-radiography Utilization Study-II (NEXUS-II) \& University of California-Davis Rule (UCDR). Of all the screening tools PECARN was the most consistent with a sensitivity of $98 \%$ and specificity $58 \%$.

\section{Conclusion}

Several screening tools have been adapted and are currently used in emergency departments to establish those children who are at risk of a TBI. While no literature was located that was specific to the out-of-hospital sector, further investigation is warranted to examine the utility of such paediatric TBI screening tools for the out-ofhospital sector.

\section{End tidal carbon dioxide in the out-of- hospital setting: Can it assist when to cease resuscitation in cardiac arrest? Claire Whitehead ${ }^{1}$, Christian Winship ${ }^{2,1}$, Brett Williams $^{2}$, Malcolm Boyle ${ }^{2}$ \\ ${ }^{1}$ ACT Ambulance Service, ACT, Australia, ${ }^{2}$ Monash University, Frankston, Victoria, Australia}

\section{Background}

The likelihood of a positive outcome from out-ofhospital cardiac arrest is extremely low; with patients found in ventricular fibrillation and receives defibrillation within 6 minutes having the highest probability of survival. A prognostic tool that could be used to guide the continuation or cessation of resuscitation efforts in the out-ofhospital setting would be useful. It has been theorised that quantitative measurement of end tidal carbon dioxide is able to guide resuscitation efforts as well as demonstrate when return of spontaneous circulation has occurred.

\section{Methods}

A literature review was undertaken using the electronic medical databases Ovid Medline, EMBASE, CINHAL Plus, Cochrane Systematic Review, and Meditext, from their commencement to the end of May 2012. Keywords used in the search included: End tidal carbon dioxide, capnography, capnometry, cardiac arrest, heart arrest, EMS, EMT, paramedic, emergency medical service, emergency medical technician, prehospital, out-of-hospital, and ambulance. References of relevant articles were also reviewed.

\section{Results}

Of the 872 articles found, 12 met the inclusion criteria. The literature was able to demonstrate that an end tidal carbon dioxide value of less than $10 \mathrm{mmHg}$ after 20 minutes of advanced life support management was predictive of extremely low likelihood of survival of out-of-hospital cardiac arrest, and almost guaranteed extremely poor neurological outcomes if patients did survive. Our literature review also was able to show that end tidal carbon dioxide has a role in predicting when a patient was about to achieve a return of spontaneous circulation.

\section{Conclusion}

From the literature reviewed, end tidal carbon dioxide is a valid tool for guiding resuscitation efforts in patients suffering from an out-of-hospital cardiac arrest and could be used as guide for when to terminate resuscitation efforts. 


\section{Pre-Employment Fitness Testing for Paramedics: an Analysis \\ Kate Thornton and Mark Sayers}

University of the Sunshine Coast, Sunshine Coast, Queensland, Australia

\section{Background}

Occupational requirements demand that paramedic professionals require moderate levels of health and fitness to carry out their duties effectively. However, current research suggests that injury and illness rates are high in the paramedic profession. Current practice uses preemployment fitness tests to screen paramedics, but questions remain as to the appropriateness of these tests and hence their effectiveness as a functional screening tool. The intent of study is to examine the reliability of a current ambulance service's pre-employment fitness test and to show whether overall test score is biased toward any particular physical capacity.

\section{Methods}

To assess whether overall scores were biased by any one capacity (i.e. flexibility, strength, endurance, etc), results from the pre-employment fitness test were examined from a cohort of 3 years of paramedic university students $(n=150)$. Analyses were based on standard logistic regression and non-parametric test procedures. Intra and inter-test reliability were assessed by having a sample of two groups of students $(n=20)$ repeating the test 2 weeks apart. Results were then compared using standard tests of reliability (Typical Error of Measurement, Coefficient of Variation [CV\%], Intraclass Correlation Coefficient).

\section{Results}

Results suggested that overall test scores were biased towards some key fitness capacities; with participants able to score 0 points in many tests and still achieve an overall pass. An apparent gender effect was also present with the key determinants of overall score being different for males and females. In addition, several of the test components (e.g. dexterity, strength) presented with relatively poor intra and inter-test reliability results $(\mathrm{CV} \%>20)$.

\section{Conclusion}

Results question the effectiveness of the preemployment fitness test. In addition, the relatively poor reliability data for several of the test components means that care should be taken to avoid over-interpreting test scores. It is recommended that the test(s) and/or scoring system be re-developed.

\section{A Combined EMT Vital Sign Survey Algorithm for EMS in Taiwan: A Student's Perspective \\ Yu-tung Chang}

Monash University, Victoria, Australia

\section{Background}

In Taiwan, there are about 13,500 Emergency Medical Technician (EMT)-Firefighters and 20 different local fire departments that serve 23 million people; and is overseen by the National Fire Agency (NFA). The EMS protocol proposed by NFA only provides a basic concept of clinical skills for EMTs; therefore, instructors teach algorithms in regards to different EMS jurisdictions or their personal experiences. The lack of consistency in patient approach algorithm was experienced by the author during several clinical placement visits whilst undertaking the Bachelor of Emergency Health (Paramedics) at Monash University. This paper will outline how this lack of consistency has worsened and confused EMTs in practicing emergency health care in Taiwan. Based on this, a new algorithm developed by the author will be proposed.

Rather than using one generic patient approach, there are four basic patient approach algorithms currently being used in Taiwan EMS. These include: medical, trauma, witnessed and unwitnessed out-of-hospital cardiac arrest (OHCA). These algorithms aim to outline the basic steps in patient management for EMTs; however, the inconsistency and complicated nature of the algorithms, is compromising and confusing EMTs. Therefore, a new simplified combined algorithm attempts to simplify the learning process and patient approach has been developed. Furthermore, a new concept of ALS case checking tool, in which the ALS case criteria are allocated, will be introduced to assist this combined algorithm at the step of ALS case determination.

\section{Conclusions}

It is hoped that this simplified patient approach algorithm has the capacity to assist EMTs to identify elements and steps in the processes of patient management. Moreover, it also has the capacity to perform a systematic examination and treatment in out-of-hospital settings that currently appears not to be occurring. This algorithm will require further study to determine its efficacy in EMS in Taiwan. 


\section{The capacity of NSW paramedics to recognise septic patients with a screening tool (PASTa study).}

Ric Thomas

Ambulance Service of NSW, Sydney, New South Wales, Australia

\section{Background}

In NSW, sepsis and septic shock have been identified as preventable causes of morbidity and mortality. Many septic and severely infected patients make their way into the health system by ambulance and studies have shown that prehospital sepsis recognition and care improves the time to definitive treatment. This study had 2 Background: 1. To assess existing paramedic capacity to identify patients with, or at risk of, sepsis; 2 . To assess the efficacy of a prehospital sepsis screening tool in improving the ability of paramedics to identify patients with, or at risk of, sepsis.

\section{Methods}

A sepsis recognition score (SRS) was devised by the researchers to assess paramedic capacity to identify patients with, or at risk of, sepsis. Participants for the study were randomly selected from employee records of practicing Ambulance Service of NSW paramedics. Voluntary participation and incentives were offered. Objective 1 was met via an online survey comprising 10 case studies; the primary outcome was the per cent of patients correctly identified as having, or at risk of having, sepsis. Objective 2 was met via a randomised controlled trial; the primary outcome was mean difference in SRS.

\section{Results}

Of the 900 randomly selected invitees, 241 volunteered to participate with 148 completing Part A and 115 completing Part B. Analysis is underway and full results will be reported.

\section{Conclusions}

This study will contribute to an understanding of the capacity of paramedics within NSW to recognise patients with sepsis. Further, it will quantify the discriminative value of a tool designed to assist with detection of sepsis, and make recommendations for future studies.

\section{Victorian Ambulance Cardiac Arrest Registry (VACAR): Over a Decade of Results from Victoria, Australia.} Karen Smith ${ }^{1,3}$, Peter Cameron ${ }^{2}$, Stephen Bernard $^{1,2}$, Ziad Nehme ${ }^{1,2}$, on behalf of the VACAR Steering Committee

\footnotetext{
${ }^{1}$ Ambulance Victoria, Doncaster, Victoria, Australia ${ }^{2}$ Monash University, Prahran, Victoria, Australia,

${ }^{3}$ University of Western Australia, Perth, Western Australia, Australia
}

\section{Background}

The American Heart Association states that the treatment of out-of-hospital cardiac arrest (OHCA) by Emergency Medical Services (EMS) be the sentinel measure of the quality of EMS care in the community.

\section{Methods}

The Victorian Ambulance Cardiac Arrest Registry (VACAR) is funded by the Department of Health and contains information dating back to 1999 , for all patients in Victoria, Australia, who suffer an OHCA and receive an ambulance. EMS data is captured from patient care records (now electronic) and operational databases. Discharge data is obtained from hospital records and the state death registry. Data on aetiology is also obtained from a state coronial registry for discrete projects. VACAR has also commenced 12-month quality-of-life (QOL) follow-up using known $Q O L$ tools administered via a telephone interview.

\section{Results}

VACAR is used to inform strategy decisions, evaluate programs and support epidemiological research and clinical trials. Key strengths of the registry include the contemporary nature of the data (less than one month delay post event), limited missing data and rigorous quality control procedures. From January 2001 to December 2011 there were 48,721 cardiac arrest patients attended by ambulance, of which $41 \%$ had resuscitation attempted by EMS (average 4,429 per year). Since 2001, adult arrests where EMS attempted resuscitation $(n=19,201)$ has observed statistically significant increases in bystander CPR ( $41 \%$ in 2001 vs. $63 \%$ in 2011 ), survival to hospital (21\% vs. $32 \%)$ and survival to discharge ( $6 \%$ vs. $11 \%$ ), $p<0.001$ for all (excludes EMS witnessed OHCA). Survival to discharge in VF/VT patients has increased from $9 \%$ to $28 \%(p<0.001)$, and drives the majority of the survival improvement.

\section{Conclusion}

VACAR is one of the largest cardiac arrest registries in the world and has been used effectively by Ambulance Victoria to drive improvements in patient outcomes from OHCA. 


\section{Trans-electrical nerve stimulation (TENS) for acute pain management in the prehospital setting: a systematic review and meta-analysis of randomised controlled trials.}

Paul M Simpson, Ric E Thomas, Pieter F

Fouche, Jason C Bendall

Australasian College of Ambulance Professionals (NSW), Sydney, New South Wales, Australia

\section{Background}

To undertake a systematic review to determine the effectiveness of paramedic-performed TENS for providing analgesia and anxiolyis in the prehospital setting, and to meta-analyse results from homogenous studies.

\section{Methods}

A systematic review was conducted to identify randomised controlled trials performed in the prehospital setting comparing 'real' TENS with 'sham' TENS for managing acute pain, with outcomes measured using a visual analogue scale (VAS). Systematic electronic searches were conducted of key databases (Medline, CINAHL, EMBASE, and Cochrane Library). Assessment of validity and bias was conducted using validated tools. A meta-analysis was performed using a random-effects model to determine the difference in mean final posttreatment VAS score for the primary outcome of pain severity and secondary outcome of anxiety.

\section{Results}

There were 4 randomised controlled trials that met the inclusion criteria. All were methodologically sound and of good quality. Initial pain and anxiety levels were similar for control and intervention groups in all studies. Aetiology of acute pain differed across studies including pelvic pain in women, traumatic hip pain in older patients, traumatic back pain, and renal colic. All studies found patients who received 'real' TENS to have significantly lower final pain and anxiety scores compared to 'sham' TENS. The reductions in pain score in those who received real TENS were clinically significant in magnitude. There were no serious adverse effects reported. In the meta-analysis of 4 studies, patients with real TENS had a final post-intervention VAS score $32 \mathrm{~mm}(95 \% \mathrm{Cl} 44-21 ; p<0.0001)$ lower for acute pain and $26 \mathrm{~mm}(95 \% \mathrm{Cl} 35-17)$ lower for acute anxiety, than those receiving 'sham' TENS.

\section{Conclusion}

Paramedic-performed TENS is an effective prehospital intervention which provides clinically significant pain relief and anxiolysis. TENS should be considered as a prehospital analgesic option in systems or circumstances in which pharmacological analgesia is infeasible or unavailable.

\section{Long term pain prevalence and health related quality of life outcomes for patients enrolled in a ketamine versus morphine for prehospital traumatic pain randomized controlled trial}

Paul Jennings ${ }^{1,2}$, Peter Cameron ${ }^{3,4}$, Stephen Bernard ${ }^{2,3}$, Tony Walker ${ }^{2}$, Damien Jolley ${ }^{3}$, Mark Fitzgerald ${ }^{3}$, Kevin Masci ${ }^{2}$

${ }^{1}$ Monash University, Department of Community Emergency Health and Paramedic Practice, Melbourne, Victoria, Australia

${ }^{2}$ Ambulance Victoria, Melbourne, Victoria, Australia

${ }^{3}$ Monash University, Department of Epidemiology and Preventive Medicine, Melbourne, Victoria, Australia

${ }^{4}$ The Alfred Hospital, Melbourne, Victoria, Australia

\section{Background}

There is some data that improved early pain control impacts on the longer term incidence of persistent pain. In a randomized, controlled trial, we found that the administration of ketamine decreased pain scores at hospital arrival compared with morphine in patients with prehospital traumatic pain. In this follow-up study, we sought to determine the incidence of persistent pain and whether there were differences in patients who received ketamine or morphine.

\section{Methods}

This study was a long-term follow-up study of the prehospital, prospective, randomized, controlled, open-label study comparing ketamine with morphine in patients with trauma and a verbal pain score of $>5$ after $5 \mathrm{mg}$ IV morphine. Patients were followed up by telephone six to twelve months following enrolment and questioned using the SF36 (version 2) health related quality of life survey and the verbal numerical rating pain scale.

\section{Results}

A total of $97 / 135(72 \%)$ patients were able to be followed up six to twelve months following enrolment between July 2008 and July 2010 . Overall, 44/97 (45\%) participants reported persistent pain related to their injury, with $3 / 97$ (3\%) reporting persistent severe pain. The prevalence of persistent pain was the same between study groups (ketamine group: 22/50, $44 \%$ compared with the morphine group: $22 / 47$, $46 \%$ ). There was no difference in the SF-36v2 scores between study arms.

\section{Conclusion}

There is a high incidence of persistent pain after traumatic injury. Although ketamine has decreased pain scores at hospital arrival compared with morphine, this difference does not impact on the prevalence of persistent pain after 6 months. Further larger studies are required to confirm this finding. 


\section{Describing Pain Following Road Trauma: Predictors of Persistent Pain and Pain Prevalence \\ Paul Jennings ${ }^{1,2}$, Belinda Gabbe ${ }^{5}$, Pam \\ Simpson $^{3}$, Karen Smith ${ }^{1,4}$, Peter Cameron ${ }^{5}$ \\ ${ }^{1}$ Monash University Department of Community \\ Emergency Health and Paramedic Practice, Melbourne, Victoria, Australia \\ ${ }^{2}$ Ambulance Victoria, Melbourne, Victoria, Australia \\ ${ }^{3}$ Monash University Biostatistics Consulting Service, Melbourne, Victoria, Australia \\ ${ }^{4}$ University of Western Australia Discipline - Emergency \\ Medicine, Perth, Western Australia, Australia \\ ${ }^{5}$ Monash University Department of Epidemiology and \\ Preventive Medicine, Melbourne, Victoria, Australia}

\section{Background}

To describe the prevalence of pain at 6 and 12 months following road trauma, and to identify predictive factors for persistent pain in this group.

\section{Methods}

This cohort study reports on 2,246 patients who sustained transport related injury between June 2007 and December 2009. Data were extracted from the Victorian State Trauma Registry and linked with the Ambulance Victoria clinical information system to obtain prehospital data. Pain scores were obtained from prehospital data and collected by telephone interview at 6 and 12 month post injury.

\section{Results}

The majority of those who sustained road trauma were male $(72 \%)$ and young (median age 39 [IQR 25-55] years). Most of those injured were motor vehicle drivers or passengers $(54 \%)$. Over half $(53 \%)$ of those injured reported pain 6 months post injury and $48 \%$ reported pain at 12 months. Females were more likely to experience persistent pain (AOR 1.5; 95\% Cl 1.1-2.1). Compared to 18 and 19 year olds, older people had greater odds of experiencing pain, especially those in the 40-59 year age group (AOR 3.6; 95\% Cl 1.9-6.9). Compensable patients demonstrated increased odds of reporting persistent pain (AOR 3.9; 95\% $\mathrm{CI}$ 2.4-6.4) and the presence of pain on arrival at an ED was associated with greater odds of experiencing pain at 12 months (AOR 1.7; $95 \% \mathrm{Cl}$ 1.0-2.7). Compared to isolated orthopaedic injury, patients with isolated head injury (AOR 0.3; 95\% $\mathrm{Cl}$ 0.2-0.6) or chest / abdominal injuries (AOR 0.6; $95 \% \mathrm{Cl} 0.4-1.0$ ) were less likely to report pain 12 months following injury.

\section{Conclusion}

Persistent pain at 6 and 12 months following transport related injury was prevalent. Several factors have been identified which appear to be associated with an increased likelihood of having persistent pain 12 months following injury.

\section{Should intraosseous access be the first choice of access for ambulance services when attending patients in cardiac arrest? Simon Allison ${ }^{1,2}$ and Alan Wilson ${ }^{1,2}$}

${ }^{1}$ Whitireia, Wellington, New Zealand
${ }^{2}$ Wellington Free Ambulance, Wellington, New Zealand

\section{Background}

Intraosseous (IO) access is a potential route for drug administration in cardiac arrest. Paramedics can reliably obtain 10 access, and complication rates are low. This study set out to test the hypothesis that $I O$ access can be gained faster, give better drug distribution and improve cardiac arrest outcomes when compared to intravenous (IV) access and should therefore be the first choice of drug access for paramedics when attending patients in cardiac arrest.

\section{Method}

A systematic literature review was conducted using the electronic databases: Pubmed; Sciencedirect; Proquest; and the Cochrane Library. Inclusion criteria were studies comparing tibial intraosseous (tIO) with peripheral intravenous (pIV) access that measured either cardiac arrest outcome, drug distribution or time to gain successful access. Literature reviews, guidelines and expert opinion articles on this subject were also included. 23 unique results were located, and one further review was manually included.

\section{Results}

Studies of time to successful access $(n=2)$ showed the tIO and pIV routes to be comparable. Most studies of drug distribution and effect $(n=3 / 4)$ found tIO comparable to or better than pIV. One study showed a marked improvement in cardiac arrest survival measures for immediate adrenaline given tIO compared with delayed pIV. Insufficient evidence was found to prove the superiority of either tIO or pIV access over the other in all cases. Guidelines and expert opinion recommend IO be utilised immediately or with minimal delay in children; for adults, most guidelines reserve IO for use when IV has failed, although one expert opinion article supports immediate use.

\section{Conclusion}

We recommend that $\mathrm{IO}$ access be clearly included in ambulance service cardiac arrest guidelines and that appropriately trained paramedics be allowed to use IO as a first-line option for drug access in cardiac arrest when IV access is likely to be delayed. 


\section{Air Versus Oxygen In myocarDial infarction (AVOID) study: trial methods and experience from initial randomisations.}

Karen Smith ${ }^{1,3}$, Ziad Nehme ${ }^{1,2}$, Dion Stub ${ }^{4,5}$, Michael Stephenson ${ }^{1}$, Stephen Bernard ${ }^{1,2}$, on behalf of the AVOID Steering Committee

${ }^{1}$ Ambulance Victoria, Doncaster, Victoria, Australia

${ }^{2}$ Monash University, Prahran, Victoria, Australia

${ }^{3}$ University of Western Australia, Perth, Western

Australia, Australia

${ }^{4}$ Baker IDI Heart and Diabetes Research Institute, Prahran, Victoria, Australia

${ }^{5}$ Alfred Hospital, Prahran, Victoria, Australia

\section{Background}

The role of routine supplemental oxygen for patients with uncomplicated acute myocardial infarction is questionable with conflicting data on the effects of hyperoxia. The 'Air Verses Oxygen In myocarDial infarction' or AVOID Study is a multi-centre RCT designed to determine whether withholding supplemental oxygen in normoxic patients with acute ST-elevation myocardial infarction (STEMI) prior to reperfusion decreases infarct size. The study is being conducted by Ambulance Victoria in collaboration with $\mathrm{PCl}$ capable hospitals.

\section{Methods}

AVOID will enrol 490 adult patients transported by ambulance with acute chest pain $<12$ hours duration with ST-elevation indicative of STEMI. Exclusions include: altered conscious state, prior oxygen administration, or hypoxia on arrival. Patients are randomised prehospital to either $100 \%$ oxygen via facemask or room air. The primary end-point for the study is infarct size ascertained by mean peak and area under the curve analysis for troponin and creatine kinase. Patients will also receive a cardiac MRI 6-months post infarction. A key aspect of AVOID is the immediate withholding of oxygen in the prehospital environment, which has not been a component of previous trials examining oxygen in acute myocardial infarction to-date.

\section{Results}

AVOID commenced enrolling patients transported to three hospitals in October 2011. To date, 182 patients have met study criteria of which 162 were randomised (52\% oxygen and $48 \%$ room air). Thirty six randomised patients were excluded, including patients transported to non-study hospitals $(n=12)$ and suspected STEMI patients stood down in the emergency department $(n=17)$. Final hospital diagnosis in followed-up cases todate include 77 STEMI and 17 with other diagnoses. The positive predictive value of paramedic suspected STEMI being transferred to the cardiac catheter laboratory is $85 \%$.

\section{Conclusion}

Ambulance Victoria have successfully implemented AVOID with a current enrolment rate of $91 \%$ of eligible patients. The trial is expected to take 18-24 months to recruit all patients. 


\section{Conference Poster Abstracts}

Are Australian paramedics prepared for intimate partner violence? A pilot study Simon Dousek ${ }^{1}$, Vanita Parekh ${ }^{2}$, Angela Williams $^{3}$, Brett Williams ${ }^{1}$

${ }^{1}$ Monash University, Victoria, Australia

${ }^{2}$ Forensic and Medical Sexual Assault Care, Canberra, Australia

${ }^{3}$ Victorian Institute of Forensic Medicine, Victoria, Australia

\section{Background}

Intimate partner violence (IPV) is often

encountered by paramedics, many of whom have no specific training for responding to such cases. Research has shown that paramedics are often the first and in some cases the only agency which deal with IPV victims in the out-of-hospital setting, and thus have a unique opportunity to increase screening and reporting in order to better understand and combat IPV. However there is little formal training in managing IPV for most Australian paramedics. To evaluate the level of basic knowledge and preparedness for IPV cases in a cohort of Australian paramedics.

\section{Methods}

A selection of 50 paramedics from Canberra was provided with a 2 hour workshop on IPV and sexual assault from ACT health over the period September to November 2011. Each participant completed a paper-based questionnaire before the training to assess their baseline knowledge.

\section{Results}

Ninety percent of the paramedics had encountered at least 1 case of IPV in the last year, with the average number of cases being 3.66. Only $22 \%$ reported that they felt confident managing IPV cases. Sixty six percent of participants were unaware there is no mandatory reporting of IPV in the ACT. The vast majority of participants stated that they felt additional education and training would be most helpful to improving their ability to manage IPV cases.

\section{Conclusions}

We found our participants had a poor knowledge and poor preparedness for IPV cases. There is an urgent need for additional research to be undertaken into the best methods of educating and training paramedics to appropriately respond to IPV cases. Before such studies are undertaken it would be useful to examine the baseline knowledge and preparedness of the wider Australian paramedic population.

\section{The use of child actors and video clips to enhance learner immersion in paediatric emergency simulation \\ Ann Lazarsfeld-Jensen and Amanda Hlushak}

Charles Sturt University, Bathurst NSW, Australia

Five simulations of paediatric emergencies were developed, and staged by academics at Charles Sturt University for the purpose of assessing the potential uses of simulation in inter professional education (IPE). Multiple levels of qualitative evaluation were used, including focus groups, participant feedback, video observation and de briefing. The clinical skills needed to resuscitate a child or diagnose and treat anaphylaxis are not regularly called upon in general practice, although they are the expertise of paramedics. Inter professional education aims to both transfer knowledge between health professionals, and enhance teamwork and respect between different professions that might be thrown together in emergencies. Inter professional conflict and misunderstanding contribute to errors and sentinel events, and solution strategies usually focus on team training (Harris, Treanor, et al. 2006). However paramedics and GPs do not frequently work in static teams. Simulation in medical education is an effective tool for clinical skills acquisition through repetition (McGaghie, Issenberg, Petrusa, \& Scalese, 2010), however, in IPE scenarios performance anxiety is intensified through time pressure, oppositional behaviour of actors in the role of patient family members or medical team colleagues, and unpredictable patient responses, including the death of a child. In this way medical post graduates, nursing and paramedic students can be exposed to a rare stressful event which placements cannot schedule (Adler et al., 2009). One of the realism keys in staging the simulations video clips that were viewed in a briefing before the child manikins were brought into the surgery, clothed to match the child actors. In focus groups participants affirmed that video clips heightened their immersion in the simulation. The co teaching and learning context also heightened doctors' awareness of paramedic competencies, and allowed paramedic academics and students opportunity to clarify expectations and roles when an emergency occurs in a surgery setting. 
Instant measures of immersion in high fidelity paediatric emergency simulation Ann Lazarsfeld-Jensen, Brian Haskins

Charles Sturt University, Bathurst, NSW, Australia

Several layers of qualitative data capture were used in a complex simulation of paediatric emergencies at Charles Sturt University, which aimed to explore the effectiveness of simulation in inter professional education (IPE). Doctors, nurses and paramedics participated in the series of scripted scenarios with utilised actors and high fidelity paediatric manikins. They used interactive keypads (clickers) to log instant anonymous reactions that measured immersion, which is just one element of effective simulation. Each click in response to a three minute questionnaire measured levels of anxiety, vulnerability, conflict avoidance and leadership experienced and exercised by the group in simulation. In another room using video streaming, focus groups evaluated the teaching and learning effectiveness of each scenario. IPE is a level of simulation suitable for post-graduate students whose clinical competencies may have been honed by repetitive use of manikins. The IPE scenarios gave nonemergency practitioners an opportunity to experience unusual demands on their clinical skills and decision-making. The clickers did not attempt to explore clinical competencies, although it was subject to spontaneous observation by focus group members and in later evaluation by academics. IPE introduces elements of intensity and conflict to simulation with the use of actors and pre recorded video clips of children in medical emergencies. Immersion produces the tension that stretches a practitioner to maintain professionalism at multiple levels. IPE brings together groups from various health professions to build respect, co operation and develop an exchange of skills and understanding. The clicker results showed that three of the four scenarios had produced an experience adequately immersive to validate further exploration of the inter professional elements such as respect, co operation, and the exchange of skills and knowledge between the doctors, nurses and paramedics in the simulated emergency.

\section{Suicide intervention training programmes improves the ability of first and second year paramedic students to identify and provide assistance to persons at risk. Lisa Bowerman ${ }^{1,2}$, Eve De Silva ${ }^{1}$, Paula McMullen $^{1,2}$, Christine Clifford $^{1}$ \\ ${ }^{1}$ University of Tasmania, Hobart, Tasmania, Australia \\ ${ }^{2}$ Paramedics Australasia, Tasmania Chapter, Australia}

\section{Background}

Suicide is a major public health problem with the World Health Organisation (WHO) reporting on average one million people worldwide complete suicide each year (1). In Australia in 2009, suicide was ranked the $14^{\text {th }}$ highest cause of death compared with motor vehicle crashes ranked at $20^{\text {th. }}$ (2). With these statistics it is not surprising that completed and attempted suicide is a common call out for paramedics. However historically, paramedic curriculum has focussed on education and training that provides the necessary tools to identify and manage acute medical and trauma conditions in both adult and paediatric populations, sensitive topics such as suicide and mental health problems have been inadequately addressed.

To enhance suicide awareness and improve knowledge, first and second year paramedic students enrolled in the paramedic degree at the University of Tasmania completed a 1 day suicide intervention and awareness workshop. A study was undertaken to determine the effectiveness of the workshop in improving the students' ability to identify and provide assistance to persons at risk of suicide.

\section{Methods}

The study was undertaken at the University Of Tasmania School Of Medicine. As part of the evaluation process, the participants were required to complete pre and past test questionnaires consisting of 22 items. Two questions regarding demographics were included. Quantitative responses varied from dichotomous through to those on a five point Likert scale. Descriptive statistics were run using SPSS version 19 to produce a visual display of frequencies in histogram form.

\section{Results}

The results highlighted positive differences the workshop made to the students comfort ability in talking to people about suicide and confidence in assisting people at risk of suicide.

\section{Conclusion}

The results of this study indicate education and training programmes do provide the necessary knowledge and skills required to manage 
challenging situations which is paramount in paramedic practice.

\section{Out-of-hospital Cardiac Arrest Management by First Responders: A retrospective review of an Australian Fire Fighter First Responder Program Christian Winship ${ }^{1}$, Brett Williams ${ }^{1}$, Malcolm Boyle $^{1}$, Allan Morton ${ }^{2}$}

\author{
${ }^{1}$ Monash University, Frankston, Victoria, Australia \\ ${ }^{2}$ Metropolitan Fire and Emergency Services Board, \\ Melbourne, Victoria, Australia
}

\section{Background}

Over 9,500 people die annually in Australia from sudden cardiac arrest, with strong empirical evidence suggesting early high quality CPR and early count shock being paramount for improving survival from cardiac arrest. It has also been shown that first responder programs have been able to reduce response times and increase survival rates for out-of-hospital cardiac arrest. The objective of this study was to review the management of out-of-hospital cardiac arrest by an Australian fire fighter first responder program.

\section{Method}

This study was a retrospective cohort study of all cardiac arrests attended by the Metropolitan Fire and Emergency Services Board (MFESB) over a seven-year period, March $1^{\text {st }} 2001$ to February $28^{\text {th }} 2008$, in Melbourne, Victoria.

\section{Results}

The MFESB attended 4,450 cardiac arrests. The majority of patients presented in asystole 669 $(63.7 \%)$ with just $243(23.1 \%)$ presenting in a shockable rhythm. The majority of patients in cardiac arrest were males $(64.2 \%)$ and the mean age of the patients was 67.5 years. The MFESB median response time during the study period was 5.7 minutes (IQR 2.25 minutes), range of 0.15 minutes to 31.7 minutes, which remained stable over the seven years. The rhythm on handover to paramedics was asystole in 787 $(75.1 \%)$ cases with no shockable rhythms. One in three $(31.3 \%)$ patients received bystander CPR, with a significant rise in the rate of bystander CPR occurring over the last two years.

\section{Conclusion}

This study demonstrated acceptable response times to cardiac arrests and a low bystander CPR rate prior to arrival of the MFESB. The incidence of a shockable rhythm on arrival of the MFESB was low with the main rhythm being asystole. The main rhythm on handover to paramedics was asystole with no shockable rhythms. Further research is required to determine the effect on patient outcomes.

\section{Long term pain prevalence and health related quality of life outcomes for patients enrolled in a ketamine versus morphine for prehospital traumatic pain randomized controlled trial} Paul Jennings ${ }^{1,2}$, Peter Cameron ${ }^{3,4}$, Stephen Bernard $^{2,3}$, Tony Walker ${ }^{2}$, Damien Jolley ${ }^{3}$, Mark Fitzgerald $^{3}$, Kevin Masci ${ }^{2}$

${ }^{1}$ Monash University, Department of Community Emergency Health and Paramedic Practice, Melbourne, Victoria, Australia

${ }^{2}$ Ambulance Victoria, Melbourne, Victoria, Australia

${ }^{3}$ Monash University, Department of Epidemiology and Preventive Medicine, Melbourne, Victoria, Australia ${ }^{4}$ The Alfred Hospital, Melbourne, Victoria, Australia

\section{Background}

There is some data that improved early pain control impacts on the longer term incidence of persistent pain. In a randomized, controlled trial, we found that the administration of ketamine decreased pain scores at hospital arrival compared with morphine in patients with prehospital traumatic pain. In this follow-up study, we sought to determine the incidence of persistent pain and whether there were differences in patients who received ketamine or morphine.

\section{Methods}

This study was a long-term follow-up study of the prehospital, prospective, randomized, controlled, open-label study comparing ketamine with morphine in patients with trauma and a verbal pain score of $>5$ after $5 \mathrm{mg}$ IV morphine. Patients were followed up by telephone six to twelve months following enrolment and questioned using the SF36 (version 2) health related quality of life survey and the verbal numerical rating pain scale.

\section{Results}

A total of $97 / 135$ (72\%) patients were able to be followed up six to twelve months following enrolment between July 2008 and July 2010. Overall, 44/97 (45\%) participants reported persistent pain related to their injury, with 3/97 (3\%) reporting persistent severe pain. The prevalence of persistent pain was the same between study groups (ketamine group: 22/50, $44 \%$ compared with the morphine group: $22 / 47$, $46 \%$ ). There was no difference in the SF-36v2 scores between study arms.

\section{Conclusion}

There is a high incidence of persistent pain after traumatic injury. Although ketamine has decreased pain scores at hospital arrival compared with morphine, this difference does not impact on the prevalence of persistent pain after 6 months. Further larger studies are required to confirm this finding. 


\section{Alternatives to Ambulance Service Use for Low Acuity Patients: A Multi-Organisation Collaboration to Map Primary Care Service Capacity}

Grant Russell $^{1,4}$, Joanne Enticott ${ }^{1,4}$, Jennifer Hester $^{2,4}$, Amee Morgans ${ }^{3,4}$, Leanne Boyd ${ }^{4}$, Brooke Vandenberg ${ }^{1,4}$

${ }^{1}$ Southern Academic Primary Care Research Unit, VIC, Australia

${ }^{2}$ South East Melbourne Medicare Local, VIC, Australia

${ }^{3}$ Ambulance Victoria, VIC, Australia

${ }^{4}$ Monash University, VIC, Australia

\section{Background}

Currently most Primary Health Care is delivered in General Practice (GP). However in providing emergency medical care, Ambulance Victoria's (AV) caseload includes a proportion of patients suitable for GP care. Some of these cases are managed by AV's Referral Service, which provides additional triage and links patients to alternative service providers. Under National Health Reform, Medicare Locals are being established to integrate primary care's responsiveness to local need. This project demonstrated a unique collaboration between the South Eastern Melbourne Medicare Local (SEMML), the Southern Academic Primary Care Research Unit (SAPCRU), and AV to examine pathways to after hours service provision.

\section{Method}

A standardised template was devised to audit routinely collected data on availability and demand for GP, AV, Emergency Departments (ED) and other primary care services in the SEMML region. A review of AV emergency caseload data was conducted to identify low acuity demand for ambulance services in the after hours period. A gap analysis was conducted in order to identify any gaps in after hours provision.

\section{Results}

Significant gaps in the after hours primary care provision were identified. Alternatives to ambulance care for low acuity patients were identified within the region and barriers to GP provision of care in the after hours period were identified.

\section{Conclusion}

This research demonstrates the value of multiorganisation collaboration for researchers to obtain quality data, for services to plan for strategic and evidence based delivery and for AV to create relationship links within primary health care. Future phases of this research will address collaborative strategies for catering to low acuity demand particularly in the after hours setting.

\section{Ambulance Victoria Attendance at Drowning cases 2007-2011}

Amee Morgans ${ }^{1,2}$, Shelley Cox ${ }^{1,2}$, Bernadette Matthews ${ }^{3}$, Karen Smith $^{1,4}$

${ }_{1}^{1}$ Ambulance Victoria, Doncaster VIC, Australia

${ }^{2}$ Monash University, Prahran VIC, Australia

${ }^{3}$ Lifesaving Victoria, Port Melbourne VIC, Australia

${ }^{4}$ University of Western Australia, Perth WA, Australia

\section{Background}

Death by drowning is a leading cause of accidental death internationally. In Australia, drowning is responsible for $20 \%$ of all child injury deaths. Whilst there are several studies on the epidemiology of fatal drowning, little is known about non-fatal drowning due to poor definition of these events, and limited data capture via hospital systems. Generally, drowning is defined as an incident where submersion/immersion in liquid has resulted in a primary respiratory impairment. This presentation aims to present an epidemiological analysis of all drowning cases including non-fatal drowning.

\section{Methods}

The Ambulance Victoria Data Warehouse (DW), which contains clinical and operational data on all ambulance attendances was used to examine drowning incidents occurring between 2007 and 2011. Data was extracted from DW using key case nature classifications including drowning/submersion/immersion. All ages were included and data was retrieved at a state-wide level.

\section{Results}

In the four year period there were 568 drowning cases. The majority of cases were male $(68 \%)$ and a large proportion aged $\leq 15(44 \%)$. One in four $(25.4 \%)$ had an initial GCS $\leq 9$ and $44 \%$ had an SPO2 $\leq 85 \%$. Forty-five cases were deceased at scene $(8 \%)$.

More than half of patients had basic airway management (53.2\%). Patients received IV fluids $(16.7 \%)$, pain relief $(8 \%)$, intravenous access $(27 \%)$, and spinal immobilisation (13.3\%). A small proportion had a suspected spinal injury $(3 \%)$ or fractures $(2.3 \%)$.

\section{Conclusions}

Ambulance data is a rich source of information about epidemiology and management of drowning cases. These patients were actively managed by paramedics with a range of medications and procedures being used to improve patient outcomes. This research is being conducted in collaboration with Lifesaving Victoria (LSV). Future research will map LSV and AV data to improve service provision and drowning case outcomes. 


\section{Characteristics and Outcomes of Patients Assessed by Paramedics and Not Transported to Hospital \\ Toby Keene, Megan Davis, Carol Brook}

ACT Ambulance Service, Canberra, ACT, Australia

\section{Background}

Cases in which a patient is assessed by Paramedics and not transported to hospital represent a significant clinical risk for ambulance services. While a source of risk, non-transport cases may also represent an opportunity for patients to be diverted from Emergency Departments to primary healthcare services, but only if such patients do not subsequently present to the ED. Ambulance Services rarely learn the outcome of patients who have been assessed but not transported unless they re-present to ambulance. American studies have used surveys and computerised record matching to examine the outcomes of non-transported patients. There is no published evidence examining the characteristics and outcomes of non-transported patients in the Australian context.

\section{Methods}

This is an exploratory qualitative study of adult patients assessed by Paramedics and not transported to an Emergency Department. Participants will be chosen by purposive sampling from adult English speaking patients, who have been assessed by Paramedics and not transported to hospital.

Participants will undertake telephone semistructured interviews designed to elicit information about the patient's experience 48 hours to 7 days following an episode of care. This time period was chosen in order to allow sufficient time for participants to access relevant healthcare, while limiting memory and recall issues. The interview will explore the participant's experiences following the episode of care, along with their understanding of the decision to not go to hospital by ambulance, the factors influencing that decision, and the consequences.

\section{Results}

Results are pending at this time.

\section{Conclusions}

An understanding of the outcomes of nontransported patients will help to quantify potential adverse events and the role of paramedics in diverting patients from emergency care.
The development of a Flexible Learning Stream of BHSc Paramedic for existing ambulance officers in New Zealand. Gary Strong ${ }^{1,2}$, Carmel Haggerty ${ }^{1}$, Craig Drayton $^{1}$

${ }^{1}$ Whitireia New Zealand, Wellington, New Zealand ${ }^{2}$ Wellington Free Ambulance, Wellington, New Zealand

\section{Background}

To describe the inception, development and growth of a flexible delivery of Bachelor of Health Science (Paramedic) at Whitireia New Zealand, to meet the growing demand for qualification upgrade from existing ambulance officers. To reflect on challenges, lessons learned and indicators of future trends in tertiary education in paramedicine.

\section{Methods}

The presentation will offer a retrospective narrative overview of the first three years of planning and development of the Flexible Learning Stream of BHSc Paramedic. Three themes emerge:

\section{Rationale:}

Enquiries from existing paramedics; geographical challenges; community base of NZ ambulance services; equity in access to education; registration consultation; qualification ownership.

\section{Infrastructure:}

Project plan and funding; stakeholder engagement; recognition of prior learning; staff recruitment; tutor development; pre entry programme to prepare for degree level study; technical and institutional challenges

\section{Effectiveness of flexible delivery:}

Contemporary educational methodology and mixed mode pedagogy; how flexible is flexible; value of online discussion; clinical placement challenges; operational rostering and attendance; assessment of performance; professional development; academic achievement

The value of narrative lies in offering lived experience and insights for critique and reflection. The intention is to provoke open discussion of issues raised, to contribute to the development of this and similar projects.

\section{Conclusions}

A near tenfold growth in student numbers indicates that a need is being met. An evaluative study has been approved to determine the true effectiveness of the programme. Qualitative and quantitative data from the programme will be analysed. Experiences of 'on' and 'off' campus students will be compared and presented as a case study. It is hypothesised that results will 
indicate the value of mixed mode pedagogy for paramedic education.

\section{Drowning related out-of-hospital cardiac arrests: characteristics and outcomes.} Kylie Dyson ${ }^{1,3}$, Janet Bray ${ }^{1,3}$, Karen Smith ${ }^{1,4}$, Bernadette Matthews ${ }^{2}$, Shelley Cox $^{1,3}$, Amee Morgans $^{1,3}$

${ }^{1}$ Ambulance Victoria, Doncaster VIC, Australia
2 Lifesaving Victoria, Port Melbourne, Australia
${ }^{3}$ Monash University, Prahran VIC, Australia
${ }^{4}$ University of Western Australia, Perth WA, Australia

\section{Background}

Few studies exploring drowning related out of hospital cardiac arrests (OHCAs) have followed victims from the scene through to hospital discharge. This population-based study aims to describe this cohort and their outcomes in the state of Victoria.

\section{Methods}

Cases and data were obtained from the Victorian Ambulance Cardiac Arrest Registry (VACAR). VACAR was searched for all Victorian cases of OHCA with a precipitating event of drowning attended by Emergency Medical Services (EMS) between October 1999 and December 2011.

\section{Results}

EMS attended 343 drowning related OHCA during the study period in Victoria representing $<1 \%$ of all OHCA ( $<1 \%$ in adults and $7 \%$ in children). Of the $343,65 \%$ occurred in a metropolitan location, $77 \%$ were adults , $66 \%$ were males and $78 \%$ were unwitnessed. Of the $23 \%$ of cases that were children $63 \%$ were under the age of 6 years. EMS attempted resuscitation for 155 (45\%) cases $(34 \%$ of adults and $82 \%$ of children). In cases where EMS resuscitation was attempted, $97 \%$ had received prior bystander CPR. Overall the proportion discharged from hospital alive was $3.6 \%(1.9 \%$ in adults and $9.3 \%$ in children). Among cases where EMS attempted resuscitation, $29 \%$ had pulse restored by arrival at hospital (30\% adults and $27 \%$ of children) and $8.2 \%$ were discharged from hospital alive $(5.9 \%$ adults and $11.5 \%$ of children). Most (92\%) were discharged home after lengthy hospital stay (median 25 days, range 3-141 days).

\section{Conclusions}

Drowning is an infrequent cause of out-of-hospital cardiac arrest and survival is rare. Further work is planned reviewing individual cases for scene details to explore predictors of survival in this group.

\section{Are paramedic students satisfied with simulation in their undergraduate education? \\ Chris Huggins and Brett Williams}

Monash University, Melbourne, Australia

\section{Background}

Simulation is used to develop holistic competencies within paramedic students during their education and training. The benefits of simulation are greatly affected by the actions of the educator in the application of a simulation. Also the attitude of the participants and the amount of emotional currency expended during the process is important and will affect the outcome of a simulation. These factors affect the simulation experience for the participant and have a bearing on the educational outcomes. Therefore the aim of the study is to examine paramedic students' satisfaction experiences of simulation during their education.

\section{Methods}

A prospective cross-sectional study using the Satisfaction with Simulation Experience Scale (SSES) was sampled on $2^{\text {nd }}$ and $3^{\text {rd }}$ year undergraduate paramedic students. The SSES is a standardised tool that contains 18 -items using a 5 -point Likert scale ( $1=$ strongly disagree $5=$ strongly agree).

\section{Results}

A total of ( $n=167)$ undergraduate paramedic students participated in the study. The majority of the participants were female $58.7 \%(n=98)$ and aged $<26$ years of age $80.2 \%(n=134)$. Overall findings suggest students were satisfied with their simulation experiences. At an item level the majority of students felt the 'facilitator provided constructive criticism during the debriefing' $(M=4.16, S D=0.51)$, that 'simulation tested my clinical ability $(M=4.46, S D=0.57)$, and 'the debriefing provided an opportunity to ask questions' $(M=4.50, S D=0.59)$. There was a significant difference on the item 'the simulation caused me to reflect on my clinical ability' between $2^{\text {nd }}$ and $3^{\text {rd }}$ year students mean 4.47 vs. $4.29(p=0.043)$.

\section{Conclusions}

Overall the students were satisfied that simulation is an effective learning and teaching approach. The students have identified the importance of interaction with the facilitator through the provision of constructive feedback and the role of debriefing and reflection has in developing and constructing knowledge. 


\section{A confirmatory factor analysis of the Self- Directed Learning Readiness Scale (SDLRS) \\ Brett Williams}

Monash University, Melbourne, Australia

\section{Background}

The Self-Directed Learning Readiness Scale (SDLRS) developed by Fisher, King and Tague (2001) measures readiness for self-directed learning among undergraduate healthcare students. While several exploratory factor analyses and one confirmatory factor analysis have examined the psychometric properties of the SDLRS, questions have been raised regarding the underlying latent constructs measured by the SDLRS. This objective of this study was to determine the best-fitting SDLRS factorial structure among three models published in the literature.

\section{Methods}

Data from the 3-factor 40-item SDLRS completed by 233 undergraduate paramedic students from four Australian universities (response rate of $26 \%$ ) were analysed using maximum likelihood confirmatory factor analysis. Comparison of model fit from the 40-item SDLRS was undertaken with the previously documented 4-factor 36-item and 3-factor 29-item SDLRS models.

\section{Results}

The model fit indices of the three 1-factor congeneric models with maximum likelihood analysis demonstrate that the 40-item SDLRS does not fit the data well. The best fitting model was the 4 -factor 36 -item SDLRS followed by the 3-factor 29-item SDLRS model.

\section{Conclusion}

The confirmatory factor analysis results did not support the overall construct validity of the original 40 -item SDLRS. The best model fit was achieved with the 36-item and 4-factor model. It is suggested that further psychometric examination of the SDLRS is undertaken. 